\title{
Hydrogen ion scattering from a potassium impurity adsorbed on graphene
}

\author{
M. A. Romero $\odot, *$ A. Iglesias-García, Evelina A. García, and E. C. Goldberg \\ Instituto de Física del Litoral (CONICET-UNL), Güemes 3450, S3000GLN Santa Fe, Argentina
}

(Received 16 May 2019; revised manuscript received 3 July 2019; published 23 August 2019)

\begin{abstract}
In this work we study the charge exchange process in the scattering of protons by potassium atoms adsorbed on a graphene surface in a low coverage limit. Both, the projected density of states on the alkaline atom site and the final charge states of the hydrogen projectile are calculated by considering the electronic Coulomb repulsion in the $s$-valence orbital. The inner $3 p$ and $3 s$ states of potassium are included and the local perturbations of the density matrix on the surrounding $\mathrm{C}$ atoms are also considered. The interacting systems are described by an Anderson Hamiltonian whose terms are calculated from the chemical properties of the atoms and the extended features of the graphene surface. The positive and negative ion fractions of hydrogen in the collision process are obtained from Keldysh-Green functions, which are calculated by employing the equation of motion method closed up to a second order in the atom-surface coupling term. It is found that the carbon atoms have no possibility of a direct charge exchange process in a frontal collision of the proton with the $\mathrm{K}$ adatom, and that the $\mathrm{K}-3 p$ band, broadened by the interaction with the graphene surface, provides an important source of electrons for the negative ionization of hydrogen, which is also promoted by the presence of a K-3s core state. The narrow $4 s$ and $3 p$ bands of the adsorbed potassium lead to an oscillatory dependence with the projectile incoming energy, of the probability for the three correlated charge states of hydrogen.
\end{abstract}

DOI: 10.1103/PhysRevB.100.085432

\section{INTRODUCTION}

Charge exchange between an atom and a surface constitutes a topic of relevance for broad areas of basic and applied research in Condensed Matter Physics. The understanding of the mechanisms of charge exchange occurring either in nonequilibrium processes like ion-surface collisions or in equilibrium processes, such as adsorption/desorption, is a matter of enormous interest in basic and applied research such as ion-electrode interchange in electrochemistry, the surface analysis technique low energy ion spectroscopy (LEIS), astrophysics, plasma and fusion research, surface ionization and its application in ion sources, and so forth [1-5].

Different mechanisms of charge transfer can take place in ion-surface scattering processes: the resonant charge transfer, which involves only one electron in a transition without change of energy; and the Auger process, which involves a two-electron interaction and requires an ionization energy of the projectile larger than twice the work function of the surface [6-8].

The presence of adsorbates on the surface can strongly affect the charge transfer in the ion scattering process by means of local and nonlocal effects. The local effect deals with the changes of the electronic potentials around the adatom, which results in local changes in the density matrix of the adsorbate-surface system. The nonlocal effect is related to the surface work-function change induced by the adsorbates, which modifies the relative position of the atomic and surface electronic levels.

\footnotetext{
*marcelo.romero@ifis.santafe-conicet.gov.ar
}

The alkaline atoms, when they are adsorbed on metal surfaces at small coverage, induce rather large work-function decreases due to the creation of a dipole layer on the surface [9]. This reduction in the surface work function can drastically enhance the negative ion formation, as it has been observed in the formation of negative ions of hydrogen by collision on a caesiated tungsten surface [10-12].

The collision of low energy alkali ions with alkali and halogen covered metal surfaces has been studied by several groups [13-19]. A conclusive remark from these works can be found in the phrase of Gauyacq et al. [19]: "Because of the importance of local effects, the resonant charge transfer process appears to be a rather sensitive probe of the local electronic properties of an adsorbate-substrate system."

In this work we study the resonant charge exchange process in the frontal collision of a proton with a potassium atom adsorbed on graphene. This is a possible situation for surfaces with a very low coverage of potassium atoms where the interaction between impurities can be neglected, and the problem is reduced to an atom adsorbed on a surface. The local effects caused by the adsorption of alkaline atoms will be detected by the ion projectile in an adequate collision geometry, which can classify the adatom site, this is the case of backscattering.

Graphene is a promising material for numerous technological applications [20], therefore any study of how the presence of adsorbates affects its properties means an important contribution [21-23]. It was found in previous works [24,25] that the localized electronic structure around alkaline atoms adsorbed on graphene is inherent to both the peculiarities of the electronic band structure of the graphene sheet, such as the energy gap that has a dramatic effect on the hybridization width, and the symmetry of the adatom valence orbital. 
We extend the calculation of the projected density of valence states on $\mathrm{K}$ atom already made in a previous work [25], by including the inner K-3s and K-3p orbital states. We also calculate, in this work, the local changes in the density matrix on the surrounding $\mathrm{C}$ atoms induced by the presence of the $\mathrm{K}$ adatom.

The interacting system is described by the Anderson Hamiltonian and the three possible charge states of the scattered hydrogen (neutral, positive, and negative) are calculated by considering the electronic repulsion in the $\mathrm{H}-1 s$ orbital $[26,27]$. The Keldysh-Green function technique together with the equation of motion method (EOM) closed up to a second order in the atom-surface coupling parameter, are used for this aim [27-29]. We also present results based on a simpler but less accurate approximation that disregards the electronic correlation (the spinless model), in order to extract from them a physical insight that is very difficult to perceive when correlation effects are introduced.

The Hamiltonian terms are obtained from a first principles calculation based on the bond-pair model [30]. The variation of the projectile energy level along the ion trajectory includes the short range interactions and also the one of large range due to the image potential [30]. The nonlocal effects of the adsorbate are considered through the electrostatic potential created by the individual dipoles associated with the adsorbed alkalis, which introduces an additional shift of the ion level [19].

The present manuscript is organized in the following way: in Sec. II we describe the atom-surface interacting system, Sec. III is devoted to the time dependent collision process, the calculation of Hamiltonian terms is presented in Sec. IV, the discussion of the results in Sec. V and finally, Sec. VI contains the conclusions.

\section{DESCRIPTION OF THE ATOM-SURFACE INTERACTING SYSTEM}

The system of interest in this work consists of a graphene surface with a very low coverage of potassium impurities, interacting with a hydrogen atom (the projectile in the scattering process). The single impurity Anderson model [31] provides the appropriate Hamiltonian for describing it, taking into account that the resonant mechanism of charge transfer is expected to be the most probable in this case:

$$
\hat{H}=\hat{H}_{\text {solid }}+\hat{H}_{\text {atom }}+\hat{H}_{\text {solid-atom }} .
$$

In Eq. (1), the first term, $\hat{H}_{\text {solid }}$, describes the solid surface (graphene) with the alkaline atom $(\mathrm{K})$ adsorbed on it; the second term, $\hat{H}_{\text {atom }}$, describes the hydrogen atom and the third term, $\hat{H}_{\text {solid-atom, }}$, is the interaction between the surface and the atom.

\section{A. Surface plus adatom system $\left(\hat{\boldsymbol{H}}_{\text {solid }}\right)$}

In the case of very low coverage the interaction between impurities can be neglected and the problem is reduced to an atom adsorbed on a surface. The $\mathrm{K}$ adsorbed on a graphene surface has been solved [25] by considering the three possible charge configurations of an $s$-valence atom: with zero, one, and two electrons. The extended single impurity Anderson model can be written by using the projection operator technique [32] as follows (see Ref. [27] for details):

$$
\begin{aligned}
\hat{H}= & \sum_{\vec{k}, \sigma} \varepsilon_{\vec{k}} \hat{n}_{\vec{k} \sigma}+E_{0}|0\rangle\left\langle 0\left|+E_{1} \sum_{\sigma}\right| \sigma\right\rangle\left\langle\sigma\left|+E_{2}\right| \uparrow, \downarrow\right\rangle\langle\uparrow, \downarrow| \\
& +\sum_{\vec{k}, \sigma}\left[V_{\vec{k} a}^{\sigma} \hat{c}_{\vec{k} \sigma}^{\dagger}|0\rangle\left\langle\sigma\left|+V_{\vec{k} a}^{\sigma *}\right| \sigma\right\rangle\langle 0| \hat{c}_{\vec{k} \sigma}\right] \\
& +\sum_{\vec{k}, \sigma}(-1)^{p_{\sigma}}\left[V_{\vec{k} a}^{\sigma} \hat{c}_{\vec{k} \sigma}^{\dagger}|\bar{\sigma}\rangle\left\langle\uparrow, \downarrow\left|+V_{\vec{k} a}^{\sigma *}\right| \uparrow, \downarrow\right\rangle\langle\bar{\sigma}| \hat{c}_{\vec{k} \sigma}\right], \quad(2)
\end{aligned}
$$

where $\vec{k}$ denotes the surface states of the pure graphene with energy $\varepsilon_{\vec{k}}$ and $\hat{n}_{\vec{k} \sigma}=\hat{c}_{\vec{k} \sigma}^{\dagger} \hat{c}_{\vec{k} \sigma}$ as its number occupation; $|0\rangle$, $|\sigma\rangle$, and $|\uparrow, \downarrow\rangle$ represent the electronic configurations of the impurity atom with zero electron, one electron with spin $\sigma$ (up or down), and two electrons, respectively ( $\bar{\sigma}$ represent the opposite spin to $\sigma$ ). The hopping integral $V_{\vec{k} a}^{\sigma}$ is the coupling between the $\vec{k}$-surface state and the $a$-orbital of the impurity atom. The total energies $E_{i}$ are related with the ionization energy of the impurity atom $\varepsilon_{I}$, and with the intrasite electronic Coulomb repulsion $U$ in the $s$-valence orbital, in the following way:

$$
\begin{gathered}
E_{1}-E_{0}=\varepsilon_{I}, \\
E_{2}-E_{0}=2 \varepsilon_{I}+U .
\end{gathered}
$$

In Eq. (2) the antisymmetry of the two electron wave function is taken into account through $p_{\sigma}=0$ if $\sigma=\uparrow$ and $p_{\sigma}=$ 1 if $\sigma=\downarrow$, and the norm condition of the atomic configuration subspace given by

$$
|0\rangle\left\langle 0\left|+\sum_{\sigma}\right| \sigma\right\rangle\langle\sigma|+| \uparrow, \downarrow\rangle\langle\uparrow, \downarrow|=\hat{1}
$$

is also contemplated.

The local $4 s$ density of states on the adsorbed $\mathrm{K}$ atom is calculated as

$$
\rho_{\sigma, 4 s}^{(K)}(\varepsilon)=\frac{1}{\pi} \operatorname{Im} G_{4 s, 4 s}^{\sigma}(\varepsilon) .
$$

The advanced Green function $G_{4 s, 4 s}^{\sigma}(\varepsilon)$ is obtained by using the following identity $[27,32]$ :

$$
\begin{aligned}
G_{4 s, 4 s}^{\sigma}(\omega)= & G_{\sigma}(\omega)+(-1)^{p_{\sigma}} G_{\sigma}^{c}(\omega) \\
& +(-1)^{p_{\sigma}} G_{\uparrow \downarrow}^{\bar{\sigma} c}(\omega)+G_{\uparrow \downarrow}^{\bar{\sigma}}(\omega)
\end{aligned}
$$

and the Green functions appearing in Eq. (5) use the Fourier transform of the following:

$$
\begin{aligned}
G_{\sigma}\left(t, t^{\prime}\right) & =i \theta\left(t^{\prime}-t\right)\left\langle\left\{|\sigma\rangle\left\langle\left. 0\right|_{t^{\prime}}, \mid 0\right\rangle\left\langle\left.\sigma\right|_{t}\right\}\right\rangle,\right. \\
G_{\uparrow \downarrow}^{\sigma}\left(t, t^{\prime}\right) & =i \theta\left(t^{\prime}-t\right)\left\langle\left\{|\uparrow, \downarrow\rangle\left\langle\left.\sigma\right|_{t^{\prime}}, \mid \sigma\right\rangle\left\langle\uparrow,\left.\downarrow\right|_{t}\right\}\right\rangle,\right. \\
G_{\sigma}^{c}\left(t, t^{\prime}\right) & =i \theta\left(t^{\prime}-t\right)\left\langle\left\{|\sigma\rangle\left\langle\left. 0\right|_{t^{\prime}}, \mid \bar{\sigma}\right\rangle\left\langle\uparrow,\left.\downarrow\right|_{t}\right\}\right\rangle,\right. \\
G_{\uparrow \downarrow}^{\sigma c}\left(t, t^{\prime}\right) & =i \theta\left(t^{\prime}-t\right)\left\langle\left\{|\uparrow, \downarrow\rangle\left\langle\left.\sigma\right|_{t^{\prime}}, \mid 0\right\rangle\left\langle\left.\bar{\sigma}\right|_{t}\right\}\right\rangle .\right.
\end{aligned}
$$

The \{\} symbol indicates the anticommutator and \langle\rangle represents the average over the Heisenberg state $\Phi_{0}$ that describes the interacting system. The Green functions, Eqs. (6) and (7), are calculated by using the EOM method up to a second order 
in the atom-surface coupling term $V_{\vec{k} a}^{\sigma}$ [27]. In this form, the $4 s$ density of states on the $\mathrm{K}$ atom adsorbed on a graphene surface is finally obtained [25]. In this work we also include in the calculation of the local density of states (LDOS), the inner K- $3 s$ and $\mathrm{K}-3 p$ adatom orbital states. The K-3s orbital with a large binding energy $(-32.4 \mathrm{eV}$ referred to the Fermi energy [33]) is a localized and fully occupied state far from the bottom of the valence band. On the other hand, the K-3p orbital $(-16 \mathrm{eV}$ referred to the Fermi energy $[33,21])$ lies within the graphene valence band, close to the bottom and far from the valence $\mathrm{K}-4 s$ state. The $3 p$ orbitals of the adsorbed $\mathrm{K}$ atom, also fully occupied, are broadened by the interaction with the graphene surface states, contributing in this form to the valence density of states located on the $\mathrm{K}$ atom. The partial density of K-3p states is calculated independently of the K- $4 s$ density of states, by assuming them as noncorrelated states, that is, within a spinless Anderson model [32]:

$$
\rho_{3 p_{i}}^{(K)}(\varepsilon)=\frac{1}{\pi} \operatorname{Im} G_{3 p_{i}, 3 p_{i}}^{(K)}(\varepsilon)=\frac{1}{\pi} \operatorname{Im}\left[\frac{1}{\varepsilon-\varepsilon_{p}-\sum_{\vec{k}} \frac{\left|V_{\vec{k}, 3 p_{i}}\right|^{2}}{\varepsilon-\varepsilon_{\vec{k}}-i \eta}}\right] .
$$

In Eq. (8), $\varepsilon_{p}=-16 \mathrm{eV}$ is the energy of the $\mathrm{K}-3 p$ orbital with respect to the Fermi energy of the graphene surface, and $V_{\vec{k} .3 p}$ is the coupling between the surface states and the $3 p$-orbital states. The total density of states on the $\mathrm{K}$ atom adsorbed on the graphene surface, incorporating the $3 p$-valence band, $\rho_{\sigma}^{(K)}(\varepsilon)=\rho_{\sigma, 4 s}^{(K)}(\varepsilon)+\sum_{i=x, y, z} \rho_{3 p_{i}}^{(K)}(\varepsilon)$, is calculated following the same procedure as in Ref. [25] for calculating $V_{\vec{k}, 3 p_{i}}$ in terms of the carbon-potassium atomic couplings. It is shown in Fig. 1 for the case of a hollow site adsorption, which is the most stable for alkali atoms, and located at 4.9 a.u. of distance from graphene $[22,34,35]$.

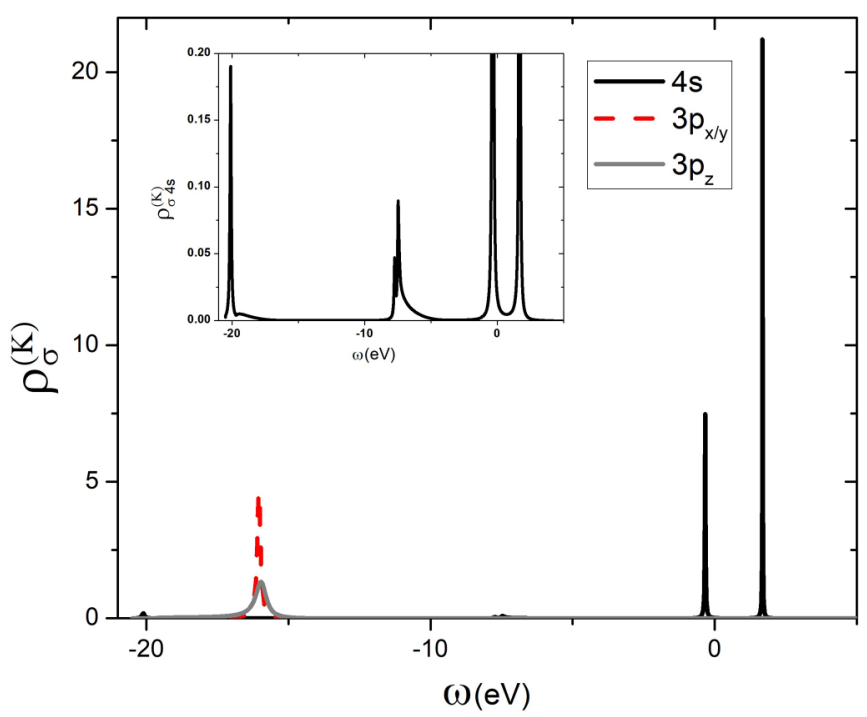

FIG. 1. Total density of states on the adatom (K). The partial contributions from the valence $4 s$ and inner $3 p \mathrm{~K}$ orbitals are indicated. The inset shows a close up view of the small $4 s$ contributions that cannot be appreciated in the main figure.
The spectral features of alkaline atoms interacting with graphene are intimately associated with the characteristics of the graphene band structure, which lead to an Anderson hybridization function [31] practically negligible except for energies close to the bottom of both the $\sigma$ and $\pi$ bands $[24,25]$. This is the origin of the two very narrow peaks near the Fermi level and of the two peaked structures observed in the K- $4 s$ density of states, one close to $-20 \mathrm{eV}$ and the other to $-7.5 \mathrm{eV}$ (see the inset of Fig. 1). The resonance of the $3 p$ orbital with the band states that occur close to the bottom of the $\sigma$ band, leads to a peak for the $3 p$ density of states wider than the peaks close to the Fermi level of the $4 s$ density of states. The differences between $3 p_{z}$ and $3 p_{x(y)}$ density of states is due to the different couplings between these K-orbital states and the orbital states of the surrounding $\mathrm{C}$ atoms. Another important thing to note is that the empty states of the potassium adsorbed on graphene are calculated by taking properly into account the electronic correlation [25].

The total occupation $n_{4 s}$ of the $4 s$-valence orbital of the adsorbed potassium at a temperature of $0 \mathrm{~K}, n_{4 s}=$ $2 \int_{-\infty}^{E_{F}} \rho_{4 s, 4 s}^{(K) \sigma}(\varepsilon) d \varepsilon$, was found to be equal to 0.9 (the Fermi level is assumed the same as for the clean surface) [25].

The local and partial density of states on the $\mathrm{C}$ sites modified by the presence of $\mathrm{K}$ adatom, is obtained from the following advanced Green function:

$$
G_{\vec{k}, \vec{k}^{\prime}}^{\sigma}\left(t, t^{\prime}\right)=i \theta\left(t^{\prime}-t\right)\left\langle\left\{\hat{c}_{\vec{k} \sigma}^{\dagger}\left(t^{\prime}\right), \hat{c}_{\vec{k}^{\prime} \sigma}(t)\right\}\right\rangle .
$$

Applying the EOM method within the Anderson model and then, Fourier transforming, one arrives to the expression (assuming the noncorrelated valence and the inner states in the adatom):

$$
\begin{aligned}
G_{\vec{k}, \vec{k}^{\prime}}^{\sigma}(\omega)= & \frac{\delta_{\vec{k}, \vec{k}^{\prime}}}{\omega-\varepsilon_{\vec{k}}-i \eta}+\sum_{a} \frac{V_{\vec{k}^{\prime} a}^{\sigma *}}{\omega-\varepsilon_{\vec{k}^{\prime}}-i \eta} \\
& \times G_{a a}^{\sigma}(\omega) \frac{V_{\vec{k} a}^{\sigma}}{\omega-\varepsilon_{\vec{k}}-i \eta},
\end{aligned}
$$

where $G_{a a}^{\sigma}(\omega)$ is the Green function of the adsorbate $(a \equiv$ $4 s / 3 p)$.

The total density of state of the surface is obtained as

$$
\rho_{\sigma}^{(S)}(\omega)=\frac{1}{\pi} \operatorname{Im} \sum_{\vec{k}} G_{\vec{k}, \vec{k}}^{\sigma}(\omega) .
$$

By replacing in Eq. (11) the expression of $G_{\vec{k}, \vec{k}}^{\sigma}(\omega)$ given by Eq. (10) and taking into account that the band states $\phi_{\vec{k}, \sigma}$, in the coupling term $V_{\vec{k} a}^{\sigma}=\left\langle\phi_{\vec{k}, \sigma}|\hat{V}| \varphi_{a}\left(\vec{r}-\vec{R}_{a}\right)\right\rangle$, can be expanded in the orthonormalized atomic basis set $\left\{\phi_{i}\left(\vec{r}-\vec{R}_{S}\right)\right\}$, as $\phi_{\vec{k}, \sigma}(\vec{r})=\sum_{i, \vec{R}_{S}} c_{i}^{\vec{k} \sigma}\left(\vec{R}_{S}\right) \varphi_{i}\left(\vec{r}-\vec{R}_{S}\right)$, Eq. (11) takes the final form

$$
\begin{aligned}
\rho_{\sigma}^{(S)}(\omega)= & \rho_{\sigma}^{(S)(0)}(\omega)+\frac{1}{\pi} \operatorname{Im} \sum_{a, i, \vec{R}_{S}, j, \vec{R}_{S^{\prime}}} V_{a, i, \vec{R}_{S}}^{\sigma *} V_{a, j, \vec{R}_{S^{\prime}}}^{\sigma} \\
& \times \int_{-\infty}^{\infty} d \varepsilon \frac{\rho_{\sigma, i, j, \vec{R}_{S}, \vec{R}_{S^{\prime}}}^{(S)}(\varepsilon)}{(\omega-\varepsilon-i \eta)^{2}} G_{a a}^{\sigma}(\omega),
\end{aligned}
$$

where $\rho_{\sigma}^{(S)(0)}$ is the total density of states, $\rho_{\sigma, i, j, \vec{R}_{S}, \vec{R}_{S^{\prime}}}^{(S)(0)}$ is the density matrix of the clean graphene surface, and 

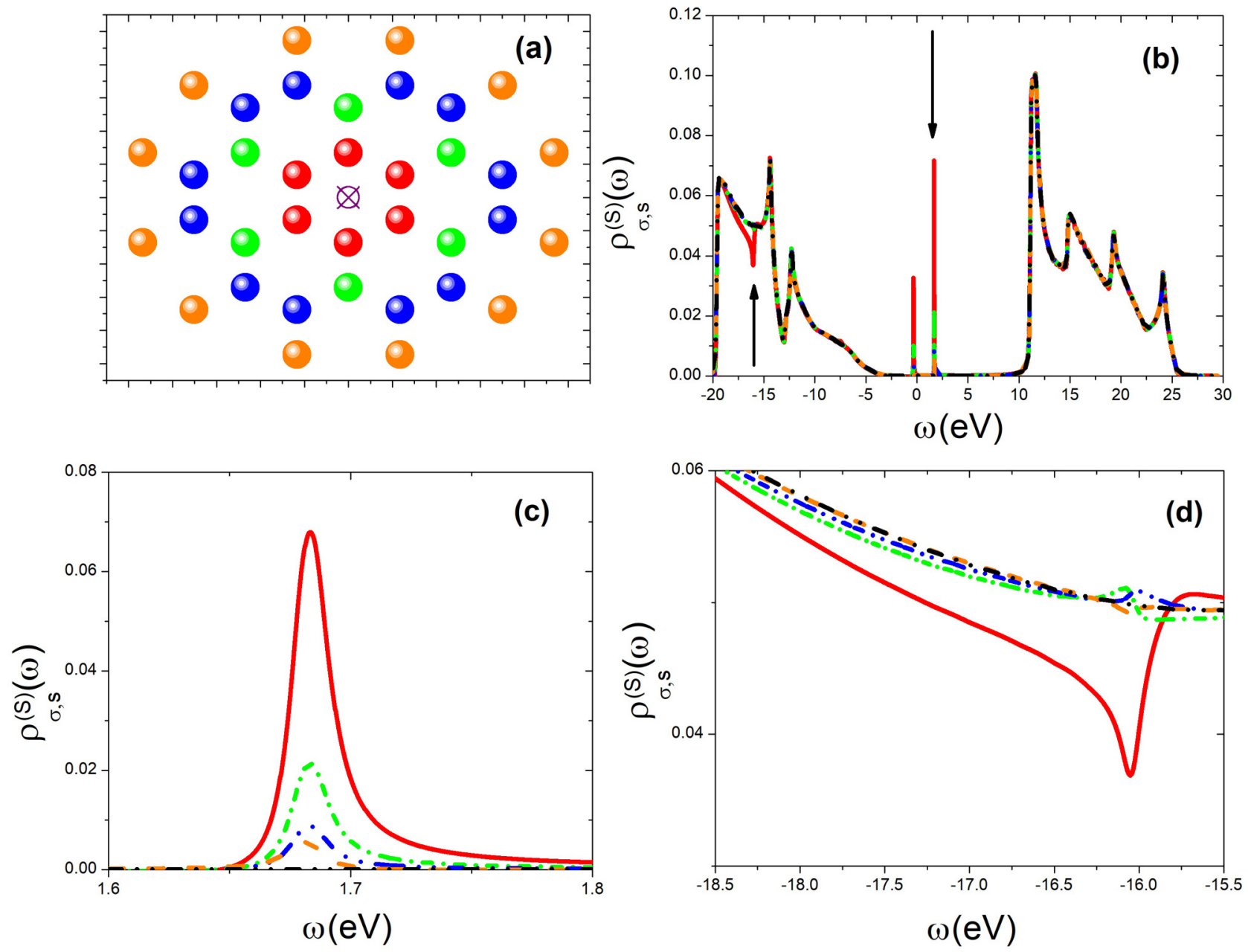

FIG. 2. (a) $\mathrm{C}$ neighbors of the $\mathrm{K}$ adatom indicated by the crossed circle. (b) Graphene partial $s$ density of states on $\mathrm{C}$ atoms at different distances from the potassium; the line color indicates the corresponding $\mathrm{C}$ atom with the same color in (a). (c) and (d) show, respectively, a larger version of the changes in the regions between 1 and $2 \mathrm{eV}$, and between -15 and $-20 \mathrm{eV}$. As reference, the local and partial $s$ density of states for the clean graphene surface is also shown (black dashed-dotted-dotted line).

$V_{a, i, \vec{R}_{S}}^{\sigma}$ is the symmetric orthonormalized hopping between the potassium $a$ state $(a=4 s, 3 p)$ and the carbon $i$ state $\left(i=2 s, 2 p_{x}, 2 p_{y}, 2 p_{z}\right)$ in the dimeric space formed by the $\mathrm{K}$ adatom and the $\mathrm{C}$ atom localized in $\vec{R}_{S}$.

From Eq. (12), we extract the local and partial graphene density of states perturbed by the potassium adatom:

$$
\begin{aligned}
\rho_{\sigma, i, i, \vec{R}_{S}, \vec{R}_{S}}^{(S)}(\omega)= & \rho_{\sigma, i, i, \vec{R}_{S}, \vec{R}_{S}}^{(S)(0)}(\omega)+\frac{1}{\pi} \operatorname{Im} \sum_{a} V_{a, i, \vec{R}_{S}}^{\sigma *} \sum_{j, \vec{R}_{S^{\prime}}} V_{a, j, \vec{R}_{S^{\prime}}}^{\sigma} \\
& \times \int_{-\infty}^{\infty} d \varepsilon \frac{\rho_{\sigma, i, j, \vec{R}_{S}, \vec{R}_{S^{\prime}}}^{(S)(\varepsilon)}(\varepsilon)}{(\omega-\varepsilon-i \eta)^{2}} G_{a a}^{\sigma}(\omega)
\end{aligned}
$$

In Figs. 2 and 3 we observe the modifications of the partial density of states on $\mathrm{C}$ atoms as the distance from the $\mathrm{K}$ adatom grows.

We can see from Figs. 2 and 3 that the perturbation produced by the presence of the $\mathrm{K}$ adatom practically disappears in the carbon fourth neighbors and that, due to the localized features of the K-LDOS, the significant changes are located in the same energy positions of the peaks found in the adatom local density of states.

As changes of lower order are expected for the off-diagonal terms of the density matrix, the following approximations are performed:

$$
\begin{aligned}
& \rho_{\sigma, i, j, \vec{R}_{S}, \vec{R}_{S^{\prime}} \neq \vec{R}_{S}}^{(S)}(\omega) \\
& =\left\{\begin{array}{l}
\rho_{\sigma, i, j, \vec{R}_{S}, \vec{R}_{S^{\prime}} \neq \vec{R}_{S}}^{(S)}(\omega), \text { when } \vec{R}_{S} \text { and } \vec{R}_{S^{\prime}} \text { refer to C atoms } \\
0, \text { when } \vec{R}_{S} \text { or } \vec{R}_{S^{\prime}} \text { refer to the } \mathrm{K} \text { atom }
\end{array} .\right.
\end{aligned}
$$

In this way, the $\vec{k}$ states of the system graphene plus potassium adsorbate are known through the density matrix elements $\rho_{\sigma, i}^{(K)}(\varepsilon)$ (Fig. 1) and $\rho_{\sigma, i, j, \vec{R}_{S}, \vec{R}_{S^{\prime}}}^{(S)}(\varepsilon)$ (the diagonal terms are shown in Fig. 2). These are the surface band states that will interact with the hydrogen ion projectile in the scattering process. 



FIG. 3. The same as in Fig. 2 for the graphene partial $p_{z}$ density of states on $\mathrm{C}$ atoms at different distances from the potassium.

\section{B. Atom system $\left(\hat{\boldsymbol{H}}_{\text {atom }}\right)$ and surface-atom interaction term $\left(\hat{\boldsymbol{H}}_{\text {solid-atom }}\right)$}

\section{Spinless model}

The atomic system (the hydrogen atom) can be described by assuming a spinless model in which there is only one active level that admits only one electron. The neutralization of the positive ion involves the ionization level while the negative ion formation from a neutral atom involves the affinity level. Both hydrogen charge fluctuations, $\mathrm{H}^{+} \leftrightarrow \mathrm{H}^{0}$ and $\mathrm{H}^{0} \leftrightarrow \mathrm{H}^{-}$, are treated independently. This is the simplest approximation of the Anderson Hamiltonian, and it works reasonably well in the case where one charge fluctuation is negligible compared with the other one. That is the case of atoms with an ionization level that resonates with the occupied surface states and a large intra-atomic Coulomb repulsion $U$ so that the affinity level resonates with the empty band states. By looking at Fig. 1, the scattering of protons (ionization level around -9 $\mathrm{eV}$ and the affinity level around $4 \mathrm{eV}$ ) by the potassium atom adsorbed on graphene seems to accomplish the abovementioned requirements for the spinless model to be a good approximation.
The spinless approximation to the $\hat{H}_{\text {atom }}$ and the $\hat{H}_{\text {solid-atom }}$ is given by

$$
\hat{H}_{\text {atom }}+\hat{H}_{\text {solid-atom }}=\varepsilon_{a} \hat{n}_{a}+\sum_{\vec{k}}\left(V_{\vec{k} a} \hat{c}_{\vec{k}}^{\dagger} \hat{c}_{a}+\text { c.c. }\right) .
$$

In Eq. (14) $\varepsilon_{a}$ corresponds to the ionization energy in the case of the proton neutralization process, and to the affinity energy in the case of negative ionization of a neutral hydrogen atom.

The spinless model, although it does not provide an accurate description of the final charge states of the ion projectile, it allows for a good understanding of the physical mechanisms involved in the charge exchange between the ion and the surface, without the complex issues introduced by the electronic correlation in the atom site.

\section{Finite-U: Correlated atom states}

The same Hamiltonian (2) used for describing the interaction of the $\mathrm{K}$ adsorbate with the graphene surface (Sec. II A) is used for describing, in this case, the interaction between hydrogen and the graphene $+\mathrm{K}$-adatom surface. The same charge configurations, with zero $(|0\rangle)$, one $(|\sigma\rangle)$, and two 
$(|\uparrow, \downarrow\rangle)$ electrons are valid for the $s$-valence hydrogen atom. Then, the $\hat{H}_{\text {atom }}+\hat{H}_{\text {solid-atom }}$ responds to the expression

$$
\begin{aligned}
& \hat{H}_{\text {atom }}+\hat{H}_{\text {solid-atom }} \\
& =E_{0}|0\rangle\left\langle 0\left|+E_{1} \sum_{\sigma}\right| \sigma\right\rangle\left\langle\sigma\left|+E_{2}\right| \uparrow, \downarrow\right\rangle\langle\uparrow, \downarrow| \\
& \quad+\sum_{\vec{k}, \sigma}\left[V_{\vec{k} a}^{\sigma} \hat{c}_{\vec{k} \sigma}^{\dagger}|0\rangle\left\langle\sigma\left|+V_{\vec{k} a}^{\sigma *}\right| \sigma\right\rangle\langle 0| \hat{c}_{\vec{k} \sigma}\right] \\
& \quad+\sum_{\vec{k}, \sigma}(-1)^{p_{\sigma}}\left[V_{\vec{k} a}^{\sigma} \hat{c}_{\vec{k} \sigma}^{\dagger}|\bar{\sigma}\rangle\left\langle\uparrow, \downarrow\left|+V_{\vec{k} a}^{\sigma *}\right| \uparrow, \downarrow\right\rangle\langle\bar{\sigma}| \hat{c}_{\vec{k} \sigma}\right] .
\end{aligned}
$$

In Eq. (15), $E_{0}, E_{1}$, and $E_{2}$ are the total energies of the electronic configurations with zero, one and two electrons, respectively, in the $1 s$ orbital of the hydrogen atom. The expansion of the $\vec{k}$ states of the solid in the atomic orbitals centered on the atoms constituting the solid includes now the potassium $4 s$ and $3 p$ states centered on the adatom position $\vec{R}_{\mathrm{K}}$. Then, the wave function that describes the graphene + potassium system can be written as

$$
\begin{aligned}
\phi_{\vec{k}, \sigma}(\vec{r})= & \sum_{\alpha=3 p, 4 s} c_{\alpha, \vec{R}_{\mathrm{K}}}^{\vec{k} \sigma} \varphi_{\alpha}\left(\vec{r}-\vec{R}_{K}\right) \\
& +\sum_{\vec{R}_{s}, \alpha=2 p, 2 s} c_{\alpha, \vec{R}_{s}}^{\vec{k} \sigma} \varphi_{\alpha}\left(\vec{r}-\vec{R}_{S}\right),
\end{aligned}
$$

where the first term gives the weight of the $\mathrm{K}$ adatom and the second, the weights from the carbon atoms of the graphene sheet. Both of these weight coefficients are related with the density matrix elements given by the expressions

$$
\begin{array}{r}
\rho_{\sigma, \alpha}^{(K)}(\varepsilon)=\sum_{\vec{k}} c_{\alpha, \vec{R}_{K}}^{\vec{k} \sigma *} c_{\alpha, \vec{R}_{K}}^{\vec{k} \sigma} \delta\left(\varepsilon-\varepsilon_{\vec{k}}\right), \\
\rho_{\sigma, \alpha, \beta, \vec{R}_{S}, \vec{R}_{S^{\prime}}}^{(S)}=\sum_{\vec{k}} c_{\alpha, \vec{R}_{S}}^{\vec{k} \sigma *} c_{\beta, \vec{R}_{S^{\prime}}}^{\vec{k} \sigma} \delta\left(\varepsilon-\varepsilon_{\vec{k}}\right) .
\end{array}
$$

The calculation of these quantities is detailed in Sec. II A and the results are shown in Figs. 1 and 2.

\section{TIME DEPENDENT COLLISION PROCESS}

The time dependence of the ion scattering from a surface is introduced by the movement of the ion projectile with respect to the surface with a finite velocity $\vec{v}$ along a defined trajectory $\vec{R}=\vec{R}(\vec{v}, t)$. A rectilinear trajectory normal to the surface and with constant velocity (in modulus) is assumed in this work. The energy loss of the projectile in the elastic binary collision with the target atom is taken into account, $E_{\text {out }}=\lambda E_{\text {in }}, \lambda$ being the kinematic energy loss factor that is equal to 0.9 for $\mathrm{H}-\mathrm{K}$.

The measured quantities in experiments of LEIS are the neutral, positive, and negative ion fractions after the scattering process took place, which are related to one, zero, and two electrons in the hydrogen $1 s$ orbital, respectively. The calculation of the single and double occupation of the projectile state is performed by using the Keldysh-Green functions technique $[28,29]$.

\section{A. Spinless model}

The mean occupation $\left\langle\hat{n}_{a}(t)\right\rangle$ gives the neutralization probability in the case of treating the charge fluctuation $\mathrm{H}^{+} \leftrightarrow \mathrm{H}^{0}$ for an incoming positive ion and the ionization level involved, while it gives the negative ion formation in the case of $\mathrm{H}^{0} \leftrightarrow$ $\mathrm{H}^{-}$for an initial neutral atom and the affinity level is the active one. In general, the procedure is based on the equation of motion of $\left\langle\hat{n}_{a}(t)\right\rangle[36]$, which leads to

$$
\begin{aligned}
\frac{d\left\langle\hat{n}_{a}(t)\right\rangle}{d t}= & -i\left\langle\left[\hat{n}_{a}(t), \hat{H}\right]\right\rangle \\
= & -\operatorname{Im} \sum_{\vec{k}} V_{\vec{k} a}^{*}(t)\left\{\int _ { t _ { 0 } } ^ { t } d \tau V _ { \vec { k } a } ( \tau ) \left[F_{a a}(\tau, t)\right.\right. \\
& \left.\left.-\left(2\left\langle\vec{n}_{\vec{k}}\left(t_{0}\right)\right\rangle-1\right) G_{a a}(\tau, t)\right] e^{i \varepsilon_{\vec{k}}(\tau-t)}\right\} .
\end{aligned}
$$

In Eq. (18) $\left\langle\hat{n}_{\vec{k}}\left(t_{0}\right)\right\rangle$ is the $\vec{k}$-state occupation at the initial time $\mathrm{t}_{0}$ corresponding to the noninteracting atom-surface system; $\left\langle\hat{n}_{\vec{k}}\left(t_{0}\right)\right\rangle$ is given by the Fermi distribution in the case of metal surfaces. Therefore, the Green functions required to calculate the mean occupation $\left\langle\hat{n}_{a}(t)\right\rangle$ are

$$
\begin{aligned}
F_{a a}(\tau, t) & =i\left\langle\left[\hat{c}_{a}^{\dagger}(t), \hat{c}_{a}(\tau)\right]\right\rangle, \\
G_{a a}(\tau, t) & =i \Theta(t-\tau)\left\langle\left\{\hat{c}_{a}^{\dagger}(t), \hat{c}_{a}(\tau)\right\}\right\rangle .
\end{aligned}
$$

The equations of motion for these Green functions result in

$$
\begin{aligned}
i \frac{d G_{a a}\left(t, t^{\prime}\right)}{d t}= & \delta\left(t-t^{\prime}\right)+\varepsilon_{a} G_{a a}\left(t, t^{\prime}\right) \\
& +\int_{t}^{t^{\prime}} d \tau \Sigma_{a}^{A}(t, \tau) G_{a a}\left(\tau, t^{\prime}\right), \\
i \frac{d F_{a a}\left(t, t^{\prime}\right)}{d t}= & \varepsilon_{a} F_{a a}\left(t, t^{\prime}\right)+\int_{t_{0}}^{t^{\prime}} d \tau\left\{\Xi_{a}^{R}(t, \tau) F_{a a}\left(\tau, t^{\prime}\right)\right. \\
& \left.+\Omega_{a}(t, \tau) G_{a a}\left(\tau, t^{\prime}\right)\right\},
\end{aligned}
$$

where the introduced self-energies are given by

$$
\begin{aligned}
& \Xi_{a}^{A}(t, \tau)=i \Theta(\tau-t) \sum_{\vec{k}} V_{\vec{k} a}^{*}(t) V_{\vec{k} a}(\tau) e^{-i \varepsilon_{\vec{k}}(t-\tau)}, \\
& \Omega_{a}(t, \tau)=-i \sum_{\vec{k}} V_{\vec{k} a}^{*}(t) V_{\vec{k} a}(\tau)\left\langle 2 \hat{n}_{\vec{k}}-1\right\rangle e^{-i \varepsilon_{\vec{k}}(t-\tau)} .
\end{aligned}
$$

\section{Energy distribution of electrons (holes) in the surface after the collision}

The analysis of the electron occupation of the surface states after the collision constitutes a very rich and complementary study for a better understanding of the exchange process. Simultaneously with the time variation of the atom state occupation, we have the time variation of the $\vec{k}$-band state occupation $\left\langle\hat{n}_{\vec{k}}(t)\right\rangle$, such that $\frac{d}{d t}\left[\sum_{k}\left\langle\hat{n}_{\vec{k}}(t)\right\rangle+\left\langle\hat{n}_{a}(t)\right\rangle\right]=0$. The energy distribution of the electrons in the surface is given by $N(\varepsilon, t)=\sum_{\vec{k}}\left\langle\hat{n}_{\vec{k}}(t)\right\rangle \delta\left(\varepsilon-\varepsilon_{\vec{k}}\right)$, and its time derivative can be calculated by means of [36]

$$
\begin{aligned}
\frac{d N(\varepsilon, t)}{d t}= & -\operatorname{Im} \sum_{\vec{k}} \delta\left(\varepsilon-\varepsilon_{\vec{k}}\right) V_{\vec{k} a}^{*}(t) \int_{t_{0}}^{t} d \tau V_{\vec{k} a}(\tau)\left\{F_{a a}(\tau, t)\right. \\
& \left.-\left[2\left\langle\hat{n}_{\vec{k}}\left(t_{0}\right)\right\rangle-1\right] G_{a a}(\tau, t)\right\} e^{i \varepsilon_{\vec{k}}(\tau-t)} .
\end{aligned}
$$


At the initial time, $N\left(\varepsilon, t_{0}\right)$ is equal to the occupied density of states of the surface: $N\left(\varepsilon, t_{0}\right)=\left[\rho^{(S)}(\varepsilon)+\rho^{(K)}(\varepsilon)\right] f_{<}(\varepsilon)$, being $f_{<}(\varepsilon)$ the Fermi function $\left(f_{<}\left(\varepsilon_{\vec{k}}\right)=\left\langle\hat{n}_{\vec{k}}\left(t_{0}\right)\right\rangle\right)$. After the collision, the energy distribution of electrons in the initially empty surface band states is calculated as

$$
N_{\text {initial empty }}^{\text {(electrons) }}(\varepsilon, t \rightarrow \infty)=N(\varepsilon, t \rightarrow \infty)\left(1-f_{<}(\varepsilon)\right),
$$

and the energy distribution of holes in the initially occupied band states is

$N_{\text {initial occ }}^{(\text {holes }}(\varepsilon, t \rightarrow \infty)=N\left(\varepsilon, t_{0}\right)-N(\varepsilon, t \rightarrow \infty) f_{<}(\varepsilon)$.

\section{B. Finite- $U$ : Correlated atom states}

In this approximation, the probability of having one electron with spin projection $\sigma$ in the atomic orbital is $n_{1 \sigma}(t) \equiv$ $\langle\mid \sigma\rangle\langle\sigma \mid\rangle_{t}$ and the probability of having two electrons is $n_{2}(t) \equiv\langle\mid \uparrow, \downarrow\rangle\langle\uparrow, \downarrow \mid\rangle_{t}$. Then, the neutralization probability is $P^{0}(t)=\sum_{\sigma} n_{1 \sigma}(t)$ and the negative ion formation probability is $P^{-}(t)=n_{2}(t)$.

The single $\left(n_{1 \sigma}\right)$ and double $\left(n_{2}\right)$ occupations are obtained from their time derivatives which, within a strict second order in the $V_{\vec{k} a}^{\sigma}$ coupling term, are given by

$$
\begin{aligned}
\frac{d n_{1 \sigma}}{d t}= & -\operatorname{Im} \sum_{\vec{k}}\left[V_{\vec{k} a}^{\sigma *}(t) \int_{t_{0}}^{t} d \tau V_{\vec{k} a}^{\sigma}(\tau)\left\{F_{\sigma}(\tau, t)-\left[2\left\langle\hat{n}_{\vec{k} \sigma}\right\rangle-1\right] G_{\sigma}(\tau, t)\right\} e^{-i \varepsilon_{\vec{k} \sigma}(t-\tau)}\right. \\
& \left.-(-1)^{p_{\bar{\sigma}}} V_{\vec{k} a}^{\sigma *}(t) \int_{t_{0}}^{t} d \tau V_{\vec{k} a}^{\sigma}(\tau)\left\{F_{\uparrow \downarrow}^{\sigma}(\tau, t)-\left[2\left\langle\hat{n}_{\vec{k} \bar{\sigma}}\right\rangle-1\right] G_{\uparrow \downarrow}^{\sigma}(\tau, t)\right\} e^{-i \varepsilon_{\vec{k} \sigma}(t-\tau)}\right], \\
\frac{d n_{2}}{d t}= & -\operatorname{Im} \sum_{\vec{k}, \sigma}(-1)^{p_{\sigma}} V_{\vec{k} a}^{\sigma *}(t) \int_{t_{0}}^{t} d \tau V_{\vec{k} a}^{\sigma}(\tau)\left\{F_{\uparrow \downarrow}^{\bar{\sigma}}(\tau, t)-\left[2\left\langle\hat{n}_{\vec{k} \bar{\sigma}}\right\rangle-1\right] G_{\uparrow \downarrow}^{\bar{\sigma}}(\tau, t)\right\} e^{-i \varepsilon_{\vec{k} \sigma}(t-\tau)} .
\end{aligned}
$$

The advanced Green functions $G_{\sigma}(\tau, t)$ and $G_{\uparrow \downarrow}^{\sigma}(\tau, t)$ that appear in Eq. (24) are the same of Eq. (6), while $F_{\sigma}(\tau, t)$ and $F_{\uparrow \downarrow}^{\sigma}(\tau, t)$ are defined as

$$
\begin{aligned}
F_{\sigma}\left(t, t^{\prime}\right) & =i\left\langle\left[|\sigma\rangle\left\langle\left. 0\right|_{t^{\prime}}, \mid 0\right\rangle\left\langle\left.\sigma\right|_{t}\right]\right\rangle,\right. \\
F_{\uparrow \downarrow}^{\sigma}\left(t, t^{\prime}\right) & =i\left\langle\left[|\uparrow, \downarrow\rangle\left\langle\left.\sigma\right|_{t^{\prime}}, \mid \sigma\right\rangle\left\langle\uparrow,\left.\downarrow\right|_{t}\right]\right\rangle .\right.
\end{aligned}
$$

These Green functions are calculated by applying the EOM method closed up to a second order in the coupling term $V_{\vec{k} a}^{\sigma}$ [27], obtaining in this form the expressions detailed in the Appendix.

\section{CALCULATION OF HAMILTONIAN PARAMETERS AND SELF-ENERGIES}

The calculation of the surface-atom coupling term $V_{\vec{k} a}^{\sigma}$ is based on an expansion of the surface states in a symmetrically orthogonalized atomic basis set, accordingly to the bond-pair model [30]. By following the procedure described in Ref. [30], one arrives at the expression

$$
\begin{aligned}
V_{\vec{k} a}^{\sigma} & =\sum_{\alpha, m} c_{\alpha, m}^{\vec{k} \sigma}\left\langle\phi_{\alpha}\left(\vec{r}-\vec{R}_{m}\right)|\hat{V}| \phi_{a}(\vec{r}-\vec{R})\right\rangle \\
& =\sum_{\alpha, m} c_{\alpha, m}^{\vec{k} \sigma} V_{\alpha m, a},
\end{aligned}
$$

where $V_{\alpha m, a}$ is the hopping integral between the atomic states $\phi_{\alpha}\left(\vec{r}-\vec{R}_{m}\right)$ of an atom of the surface and the atomic state $\phi_{a}(\vec{r}-\vec{R})$ of the projectile ion; both states belong to a symmetrically orthogonalized basis set in the dimeric space formed by the two atoms [30]. The atom-atom coupling term $V_{\alpha m, a}$ includes the electron-electron interaction within a mean field treatment [30], and the atomic wave functions for describing the atoms of interest $(\mathrm{C}, \mathrm{K}$, and $\mathrm{H}$ in this work) are obtained from Huzinaga et al. [37,38].
The $V_{\vec{k} a}^{\sigma}$ term enters in the calculation of the single and double occupations through quantities as

$$
\begin{aligned}
& \sum_{\vec{k}} V_{\vec{k} a}^{\sigma *}(t) V_{\vec{k} a}^{\sigma}(\tau) \xi\left(\varepsilon_{\vec{k} \sigma}\right) \\
& \quad=\sum_{\substack{\alpha, m \\
\beta, n}} V_{\alpha m, a}^{*}(t) V_{\beta n, a}(\tau) \int d \varepsilon \xi(\varepsilon) \sum_{\vec{k}} c_{\alpha m}^{\vec{k} \sigma *} c_{\beta n}^{\vec{k} \sigma} \delta\left(\varepsilon-\varepsilon_{\vec{k} \sigma}\right)
\end{aligned}
$$

From Eq. (27) we conclude that the complete description of the atom-surface interacting system requires knowing the localized atom-atom couplings calculated by using the bondpair model [30], and the features of the electronic band structure of the surface given by the density matrix $\rho_{\alpha m, \beta n}(\varepsilon)=$ $\sum_{\vec{k}} c_{\alpha m}^{\vec{k} \sigma *} c_{\beta n}^{\vec{k} \sigma} \delta\left(\varepsilon-\varepsilon_{\vec{k} \sigma}\right)$, whose calculation was discussed in the Sec. II A. The density matrix of the clean graphene surface is calculated by using the Fireball code based on the density functional theory and the expansion, linear combination of atomic orbitals (LCAO), of the surface states [39,40].

The variation with the distance to the surface of the one electron energy levels $\varepsilon_{I}$ and $\varepsilon_{I}+U$, is calculated from the bond-pair model without allowing charge exchange between the atom and surface. It is assumed that the short range electron-nucleus and electron-electron interactions is treated within a mean field approximation, and the effect of the long range interactions is introduced by considering the image potential defining the behavior for large normal distances to the surface [30].

It is also considered the energy level shift produced at large distances by the dipole potential formed by the adsorbate and its image [13,19,41],

$$
V_{e p}=\frac{q}{r_{1}}-\frac{q}{r_{2}},
$$




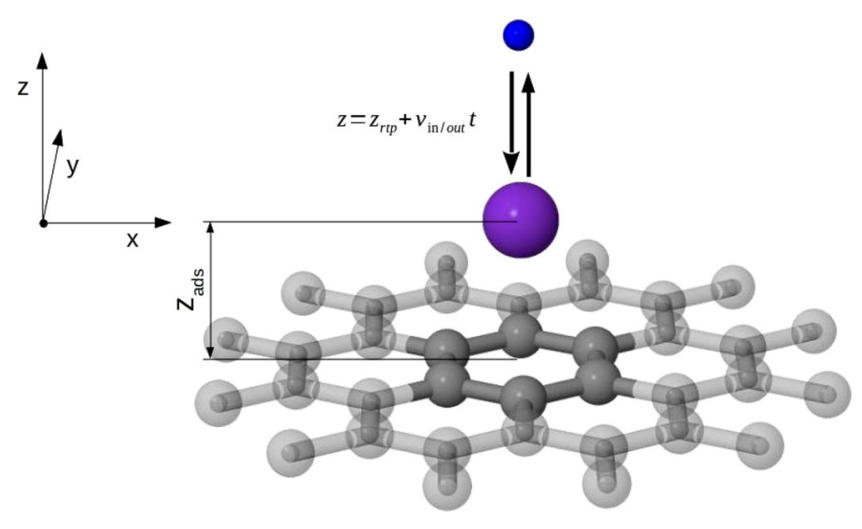

FIG. 4. Scattering geometry. The zero distance is at the $\mathrm{K}$ atom, the six $\mathrm{C}$ atoms included in the calculation are highlighted.

where $q(=0.1 e)$ is the charge of the alkaline atom [25], $r_{1}$ is the distance between the proton and the $\mathrm{K}$ adatom, and $r_{2}$ is the distance between the proton and the image charge of the positive charged $\mathrm{K}$. This potential is introduced for distances larger than 8 a.u. respect to the graphene surface, in the same way it was introduced in the image potential due to the ionic projectile.

\section{RESULTS AND DISCUSSION}

The collision geometry used in our calculation, consisting of the hydrogen scattering by the adsorbed $\mathrm{K}$ atom in a front collision, is shown in Fig. 4. The distance of closest approach at the different incoming energies is calculated consistently with the binary collision model from the interaction energy of the dimer involved, varying from 0.753 a.u. for $200 \mathrm{eV}$ to 0.176 a.u. for $2000 \mathrm{eV}$. In an attempt to better approximate the interaction energy with the $\mathrm{K}+$ graphene system, we added to the interaction energy of the dimer the interaction of the hydrogen ion with the dipole formed by the positively charged potassium and its image charge. The changes of the distances of closest approach were negligible $(0.75$ to 0.76 a.u. for $200 \mathrm{eV}$ ). The zero of distance is assumed on the $\mathrm{K}$ atom site, which is 4.9 a.u. above the graphene surface. Only the six carbon first neighbors of $\mathrm{K}$ are included in the dynamical interaction with the hydrogen projectile, based on the great distance of $\mathrm{C}$ atoms to the projectile, which leads to a negligible coupling between their states (see Fig. 6 below).

\section{A. Hamiltonian parameters}

\section{Projectile energy levels}

In Fig. 5 we show the distance evolution of the ionization and affinity levels of hydrogen, referred to the Fermi level, when the scattering is by the K adatom (see Fig. 4). The pronounced downshift observed at short distances of potassium is mainly due to the attractive electron-nucleus interaction, while the large distance behavior is determined by the dipole potential originated in the positively charged $\mathrm{K}$ and its image, Eq. (28).

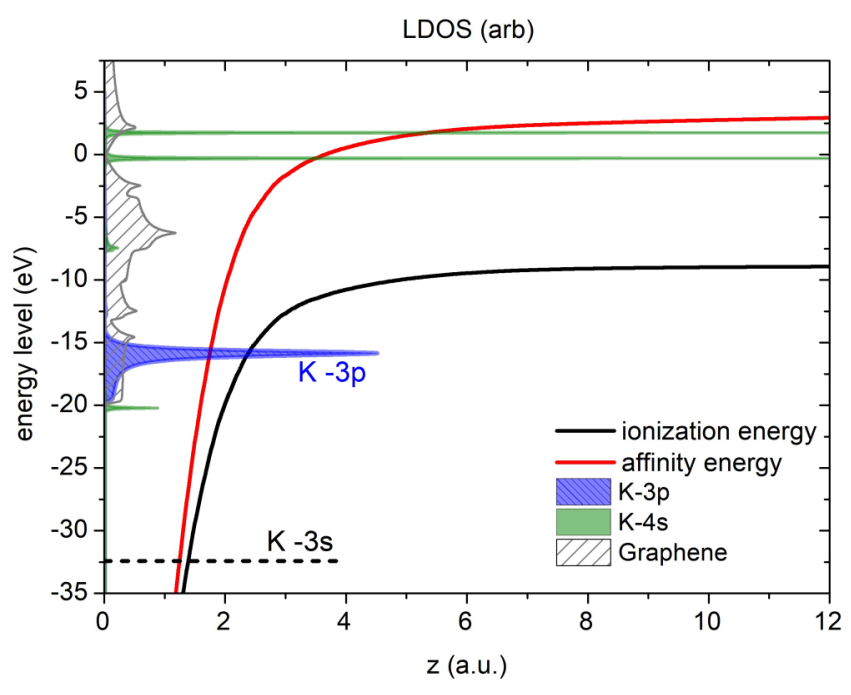

FIG. 5. The ionization and affinity energy levels of $\mathrm{H}$ as a function of the normal distance $z$ to the potassium atom. The striped area corresponds to the graphene surface LDOS and the peaked structure corresponds to the projected LDOS on the K adatom. The position of the $3 \mathrm{~s}$ core state of potassium is also indicated (dashed line).

\section{Atom-atom coupling terms}

The $\mathrm{H}-\mathrm{C}$ and $\mathrm{H}-\mathrm{K}$ coupling terms $V_{\alpha m, a}(z)$ are shown in Fig. 6 for the scattering geometry of Fig. 4. The H- $1 s$ couplings with the potassium orbital states are at least one order of magnitude larger than the couplings with the $\mathrm{C}$ states, which are practically zero at distances around 2 a.u. (note that these couplings are multiplied by 20 for being visible in the same figure). Then, from Fig. 6, we can infer that in a frontal collision of hydrogen with potassium, the interaction with the $\mathrm{C}$ atoms is negligible, justifying in this form to consider only the first 6th C neighbors of K (see Fig.4) in the LCAO expansion (26). It is effectively found that they do not

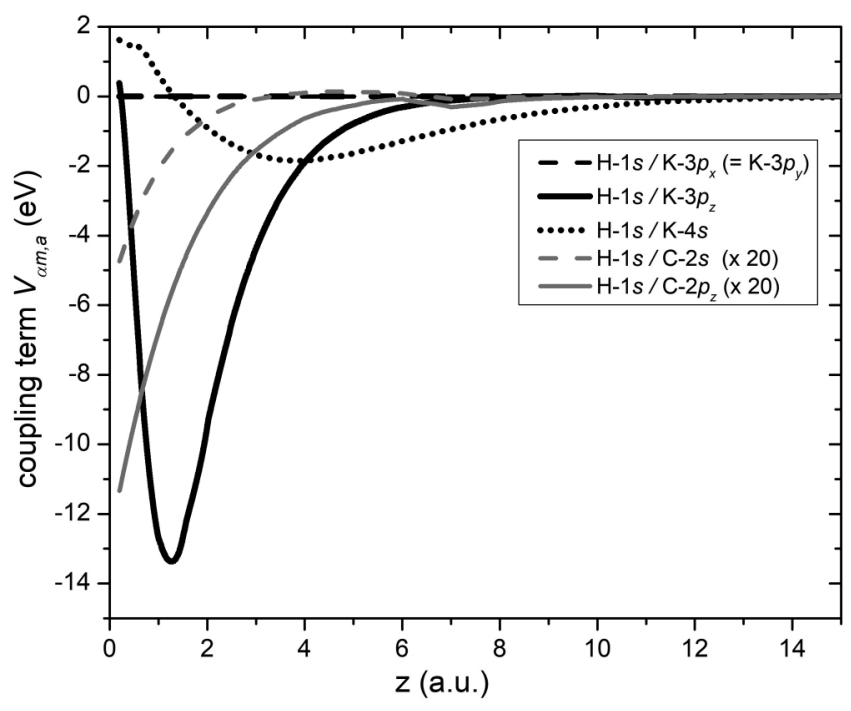

FIG. 6. Coupling terms $V_{\alpha m, a}(z)$ as a function of the normal distance to the surface $z$ for $\mathrm{H} / \mathrm{K}$ and $\mathrm{H} / \mathrm{C}$ dimeric systems (for $\mathrm{H} / \mathrm{C}$ are multiplied by 20). The zero of distances is located in the $\mathrm{K}$ atom position. 


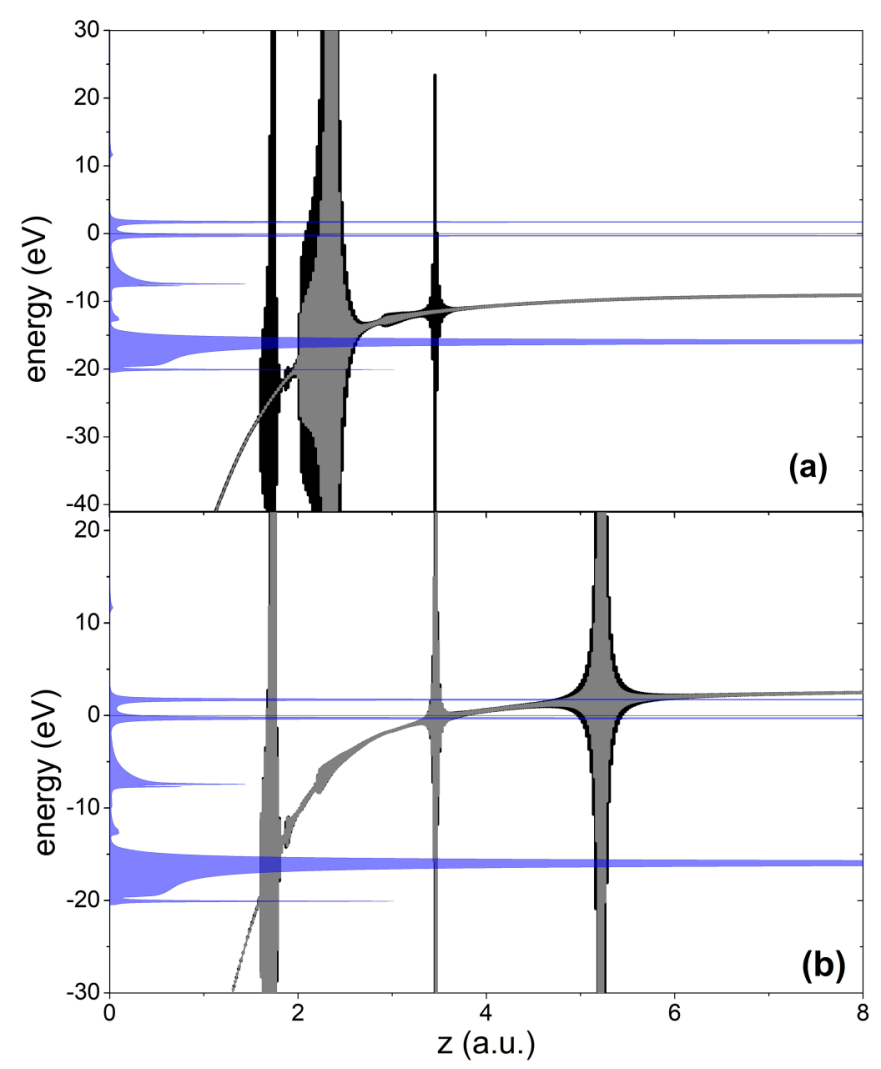

FIG. 7. The energy level and its width (see the text) shown as error bars: within an independent electron model (gray line) and by considering electronic correlation (black line). (a) Ionization level, (b) affinity level. The shadowed area corresponds to the K LDOS (the Fermi energy is set on zero).

contribute to a direct charge exchange with the ion projectile, when the alkaline adsorbate is the scattering center.

\section{Energy level widths in the spinless model}

The Anderson hybridization width is given by the imaginary part of the Fourier transform of $\Xi_{a}^{A}(t, \tau)$ [Eq. (21)], in the case of a stationary process $\left(V_{\vec{k} a}\right.$ independent of time). By introducing the LCAO expansion (26) of the $V_{\vec{k} a}$ term one arrives at the following expression:

$$
\Gamma_{0}(\varepsilon, z)=\operatorname{Im} \Xi_{a}^{A}(\varepsilon, z)=\pi \sum_{\substack{\alpha, m \\ \beta, n}} V_{\alpha m, a}^{*}(z) V_{\beta n, a}(z) \rho_{\alpha m, \beta n}(\varepsilon) .
$$

This function evaluated in either the ionization level $\left(\Gamma_{0}\left(\varepsilon_{I}, z\right)\right)$ or the affinity level $\left(\Gamma_{0}\left(\varepsilon_{I}+U, z\right)\right)$ provides an estimation of the level width due to the interaction with the surface band states in the case of disregarding the electron repulsion in the projectile atom state. In the frontal collision with the $\mathrm{K}$ adsorbate and taking into account that the $\mathrm{H}-\mathrm{C}$ coupling is practically negligible (see inset of Fig. 6), we have,

$$
\begin{aligned}
\Gamma_{0}(\varepsilon, z)= & \pi\left|V_{a, K-4 s}(z)\right|^{2} \rho_{\sigma, 4 s}^{(K)}(\varepsilon) \\
& +\pi \sum_{i=x, y, z}\left|V_{a, K-3 p_{i}}(z)\right|^{2} \rho_{\sigma, 3 p_{i}}^{(K)}(\varepsilon) .
\end{aligned}
$$

The energy levels with the respective widths as error bars, $\varepsilon_{I}(z) \pm \Gamma_{0}\left(\varepsilon_{I}, z\right)$ and $\varepsilon_{I}(z)+U(z) \pm \Gamma_{0}\left(\varepsilon_{I}+U, z\right)$, are shown in Fig. 7.

\section{Energy level widths in the finite-U model}

In the finite- $U$ calculation, another terms introduced by the correlation appear redefining $\Gamma_{0}(\varepsilon, z)$ [the imaginary parts of the Fourier transform of Eqs. (A7) in the Appendix]

$$
\begin{aligned}
\Gamma_{c}\left(\varepsilon_{I}, z\right)= & \Gamma_{0}\left(\varepsilon_{I}, z\right)+\pi \sum_{\beta=4 s, 3 p_{i}}\left|V_{a, K-\beta}(z)\right|^{2} f_{<}\left(\varepsilon_{I}\right) \\
& \times\left[\rho_{\sigma, \beta}^{(K)}\left(\varepsilon_{I}\right)+\rho_{\sigma, \beta}^{(K)}\left(\varepsilon_{I}+U\right)\right] \\
\Gamma_{c}\left(\varepsilon_{I}+U, z\right)= & \Gamma_{0}\left(\varepsilon_{I}+U, z\right) \\
& +\pi \sum_{\beta=4 s, 3 p_{i}}\left|V_{a, K-\beta}(z)\right|^{2}\left(1-f_{<}\left(\varepsilon_{I}\right)\right) \\
& \times\left[\rho_{\sigma, \beta}^{(K)}\left(\varepsilon_{I}+U\right)+\rho_{\sigma, \beta}^{(K)}\left(\varepsilon_{I}\right)\right] .
\end{aligned}
$$

These quantities are also shown in Fig. 7 as error bars mounted on the respective energy levels.

We can see that the level widths show the localized features of the local density of states on potassium, accordingly to expressions (30) and (31). The electronic correlation introduces two additional widths to the ionization level when the affinity level is below the Fermi energy and crosses the occupied K-4s and $\mathrm{K}-3 p$ density of states. It is also observed for distances between 2 and 3 a.u. that the width of the ionization level is increased when correlation effects are considered.

\section{B. Spinless calculation of the dynamical collision process}

The neutralization probability, in the case of an incoming proton, is shown in Fig. 8(a) as a function of the kinetic energy of the projectile. It is also shown the hole occupations, $\int_{\text {region } X} N_{\text {initial occ }}^{\text {(holes) }}(\varepsilon, t \rightarrow \infty) d \varepsilon$, for the different regions $X$ of the surface band structure named 1, 2, and 3 in Fig. 8(b); and the electron occupation in the initially empty band named region $4, \int_{\text {region } 4} N_{\text {initial empty }}^{\text {(electrons) }}(\varepsilon, t \rightarrow \infty) d \varepsilon$. In this form we can infer that the band states, depending on the ion velocity, largely contribute to the neutralization and reionization processes.

The ionization level as a function of the distance to the surface and its width indicated as error bars are also shown in Fig. 8(b).The first thing that draws our attention in Fig. 8(a) is the negligible electron occupation of region 4 equal to the hole occupation of region 3 in the whole energy range analyzed. This means that the electrons of the K-4s-band states close to the Fermi level are promoted to the empty $\mathrm{K}-4 s$ - band states due to the collision. Then, the reionization process takes place at the expense of the long lived holes created in regions 1 and 2 during the neutralization process. A typical exchange process, in which the electron is being transferred from narrow bands to the ion level and vice versa, seems to be occurring. Precisely, the same nonmonotonous energy dependence observed for both the hole occupation of region 1 and the electron occupation of the ionization level, for energies higher than $700 \mathrm{eV}$, is a clear manifestation of this electron exchange process, in which, depending on the ion velocity, the electron will stay either in the ion level or 

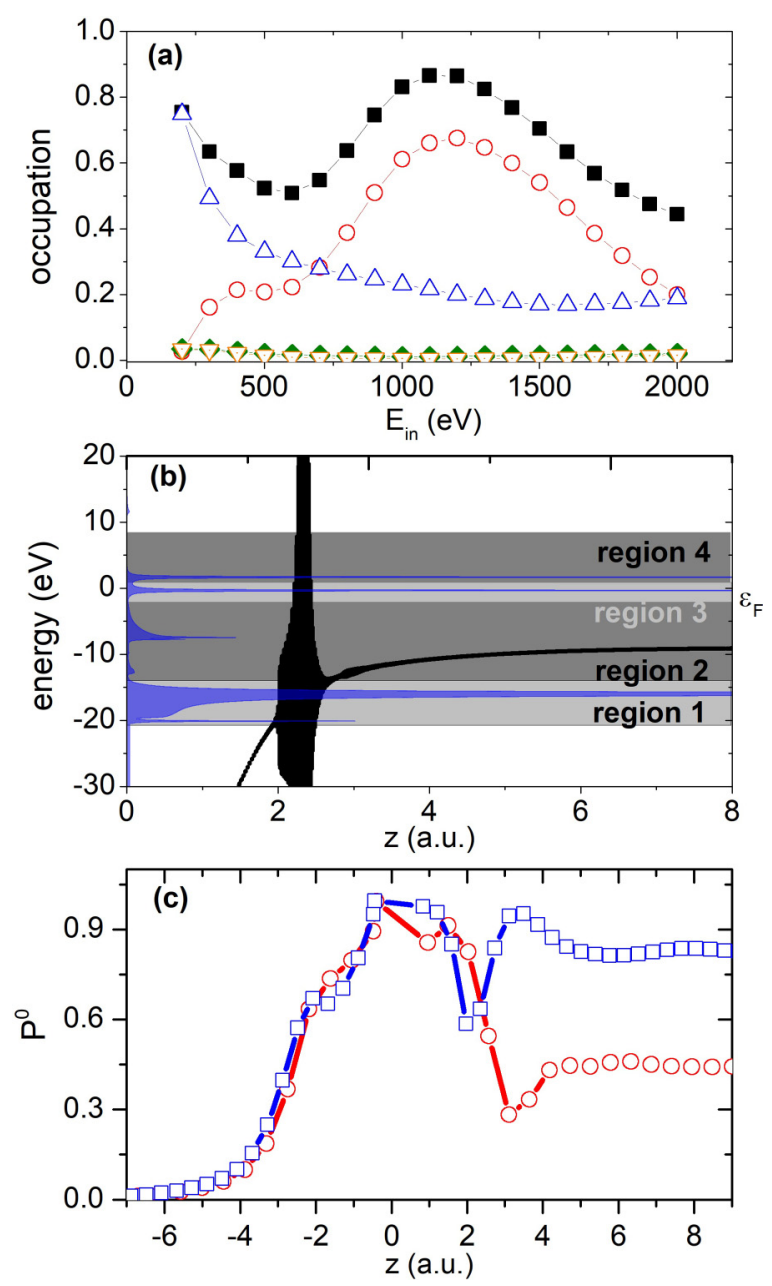

FIG. 8. (a) Neutralization probability as a function of the incoming proton energy (black full squares), hole occupation in region 1 (red empty circles), hole occupation in region 2 (blue empty triangles), hole occupation in region 3 (orange empty down triangles), electron occupation in region 4 (green full diamonds); (b) the ionization level and its width as a function of distance to the $\mathrm{K}$ adatom, the projected density of states on $\mathrm{K}$, and the shadowed areas indicate the different regions $1,2,3$, and 4 ; (c) the distance evolution (negative values of $z$ indicate the incoming path) of the neutralization probability for incoming energies $1000 \mathrm{eV}$ (blue empty squares) and $2000 \mathrm{eV}$ (red empty circles).

in the band states after the collision. The interaction with the band states of region 2 becomes more efficient as the projectile energy decreases and the effective region for the charge exchange process moves away from the surface. Figure 8 (c) shows the practically complete charge of the ionization level along the incoming path of the ion projectile, while loss and capture processes take place in the exit trajectory thanks to the holes created in the localized band states due to the neutralization during the incoming path. It is clear that the electron loss and capture processes defining the final charge state are regulated by the exit velocity. At these energies $(1000$ and $2000 \mathrm{eV})$, the participation of region 1 becomes more important through charge and discharge processes. The electron transfer from the projectile level to the holes of region 1 is more efficient as the energy is increased. Observed in
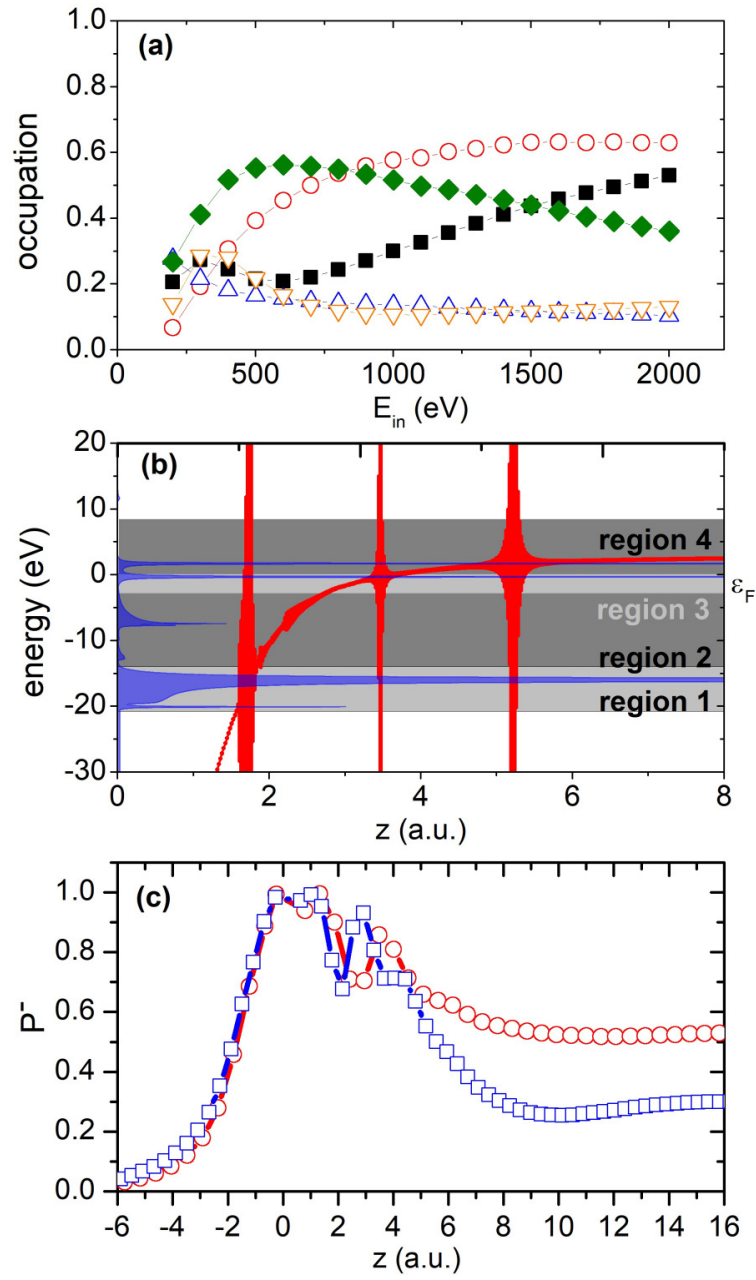

FIG. 9. (a) Negative ion formation probability as a function of the incoming neutral atom energy (black full squares), hole occupation in region 1 (red empty circles), hole occupation in region 2 (blue empty up triangles), hole occupation in region 3 (orange empty down triangles), electron occupation in region 4 (green full diamonds); (b) the affinity level and its width as a function of distance to the $\mathrm{K}$ adatom, the projected density of states on $\mathrm{K}$, and shadowed areas indicate the different regions 1,2,3, and 4; (c) the distance evolution of the negative ionization probability for incoming energies $1000 \mathrm{eV}$ (blue empty squares) and $2000 \mathrm{eV}$ (red empty circles).

Fig. 8(c), along the exit trajectory, is a discharge process followed by a charged one, at distances approximately between 2 and 3 a.u. These processes are habilitated by the interaction with the band states originated by the hybridization between the $\mathrm{K}$ and $\mathrm{C}$ states, as it is inferred from the level width observed in regions 1 and 2 of Fig. 8(b).

The bonding and antibonding interactions among the hydrogen state, the localized K-4s- and K-3p surface bands and the $\mathrm{K}-3 s$ core state, are surely playing an important role. For instance, the high efficiency of the electron exchange between region 1 and the ionization level may be favored by the bonding interaction with the K-4s band states.

On the other hand, we found that the survival probability of an incoming neutral atom is equal to 1 for all the analyzed energy values. In this form, we verified that the reionization 


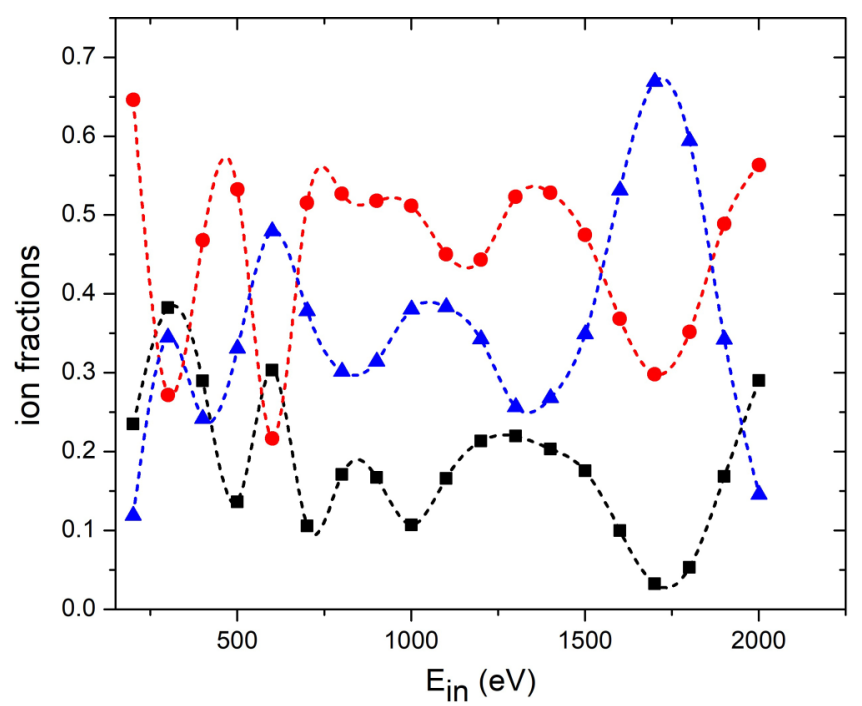

FIG. 10. Ion fractions as a function of the incoming energy: $P^{0}$ (full circles), $P^{-}$(full squares), and $P^{+}$(full triangles).

process is occurring at the expense of the holes created in the band states because of the neutralization.

From an overall view of Fig. 9(a), we can infer that the affinity level is charged at the expense of the band states of region 3,2 , and 1 , while the empty band states of region 4 are contributing to the discharge. At incoming energies below $600 \mathrm{eV}$, the charge transfer process is defined far from the surface and it is mainly produced by the interaction with the band states of region 2, 3, and 4 (the hopping with the $\mathrm{K}-3 p$ orbital decays with distance faster than the hopping with the $\mathrm{K}-4 s$ orbital (Fig. 6)]. The nonmonotonous dependence with the energy of the hole occupation of region 3, practically coincident with the electron occupation of the affinity level, is a display of the exchange process between quasiresonant localized states. At larger energy values, the distance region where the transfer process is effective becomes closer to the surface and the band states of region 1 contribute to the charge of the affinity level. At the same time, the discharge to the empty states of region 4 is less probable as the ion movement is faster, explaining in this form the increasing negative ion fraction. In Fig. 9(c), we observe that the affinity level is completely occupied in the incoming path and again, that the electron loss and capture processes defining the final charge state are regulated by the exit velocity. It is also observed in Fig. 9(c) that as the ion energy increases, the survival probability of negative ions moving away from the surface grows in the region close to the surface $(z<6$ a.u.) and the discharge to the band states of region 4 diminishes. As in Fig. 8(c), observed along the exit trajectory, is a discharge process followed by a charged one, at distances approximately between 2 and 3 a.u. These processes have the same origin: the interaction with the band states originated by the hybridization between the $\mathrm{K}$ and $\mathrm{C}$ states, as is seen by means of the level width in regions 1 and 2 of Fig. 9(b).

\section{Finite- $U$ calculation of the dynamical collision process}

To introduce the electronic correlation means that there is only one atomic orbital that admits up to two electrons. The

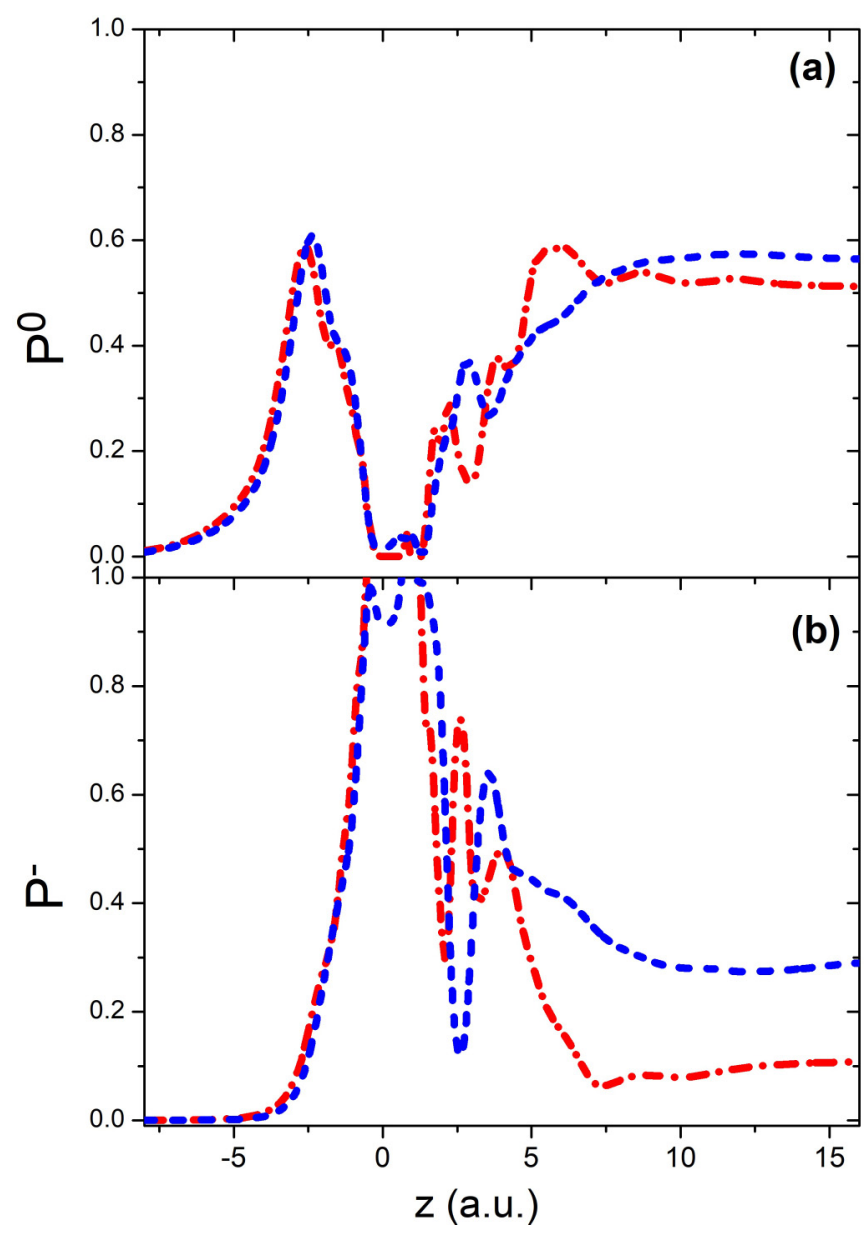

FIG. 11. Distance evolution of (a) the neutral ion fraction and (b) the negative ion fraction, for incoming energies $1000 \mathrm{eV}$ (red dashed-dotted line) and $2000 \mathrm{eV}$ (blue dashed line).

simple picture of thinking in two atomic energy levels for describing two independent processes (the ionization level for the neutralization and the affinity level for the negative ion formation) is not valid. A first signal of a correlated behavior arises from the norm condition, and in that case, if one charge state is negligible, the increasing probability of one charge state is clearly at the expense of the decreasing probability of the other. This is not occurring in our case, according to the results shown in Fig. 10, because the three charge states have an appreciable occurrence probability in the whole analyzed range of incoming energies.

It is observed in Fig. 10 that the negative ion fraction is between 10 and $40 \%$ for energies below $1500 \mathrm{eV}$, and for energies below $500 \mathrm{eV}$ the negative fraction is larger than the positive one.

In Fig. 11 we present the evolution along the trajectory of $P^{0}$ and $P^{-}$for two different energies, 1000 and $2000 \mathrm{eV}$. From Figs. 11(a) and 11(b) we observe that the proton is negatively charged along the incoming path since it has now the possibility of capturing up to two electrons. In the exit trajectory, electron loss and capture processes involving the holes created in the incoming path occur, and each charge fluctuation is accompanied by a change of the system energy. That means: in the first step, the electron loss from $\mathrm{H}^{-}$involves the 


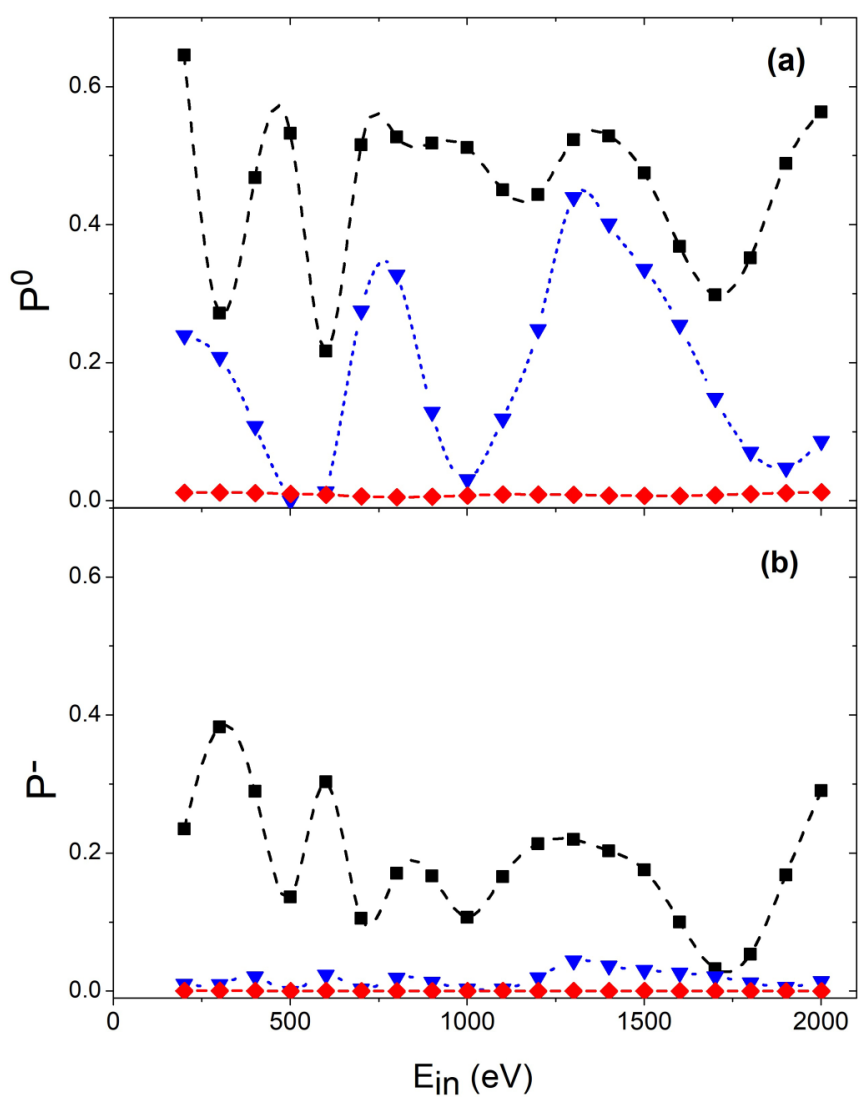

FIG. 12. (a) Neutral ion fraction and (b) negative ion fraction as a function of incoming energy. The calculation including the inner and valence states of $\mathrm{K}$ adatom (black full squares) and the calculation considering only the $4 s$-band states (blue full down triangles). Full circles (red) show the negligible contribution of the carbon atoms because they are very far from the projectile.

affinity level, but in the following step, the electron loss from $\mathrm{H}^{0}$ involves the ionization level. This correlated behavior is possibly responsible for such an oscillatory dependence on the energy of the ion fraction, compared with the noninteracting spinless calculation [Figs. 8(a) and 9(a)].

The loss processes in the empty $4 s$-band states also occur, but in this case they are habilitated not only from the affinity level but also from the ionization one, as it is suggested by its larger width in the case of correlated charge channels [Fig. 7(a)].

The important role of the inner $3 p$ and $3 s$ states of potassium can be inferred by omitting them in the calculation of the dynamical charge transfer process. In Fig. 12 we compare the calculation including the core state $3 s$ and the valence band states $3 p$ and $4 s$ of the potassium adsorbed on graphene, with the calculation that includes only the $\mathrm{K}-4 s$ band states. It is found that the negative ion formation is strongly diminished when the inner states of $\mathrm{K}$ are not considered, and a reduction, but not so marked, of the neutralization probability is also observed. The more defined oscillatory behavior with energy of $P^{0}$ seems to be related to the typical interaction between two localized states.

In Fig. 12, also shown is the negligible contribution of the $\mathrm{C}$ atoms of the graphene surface to the charge exchange

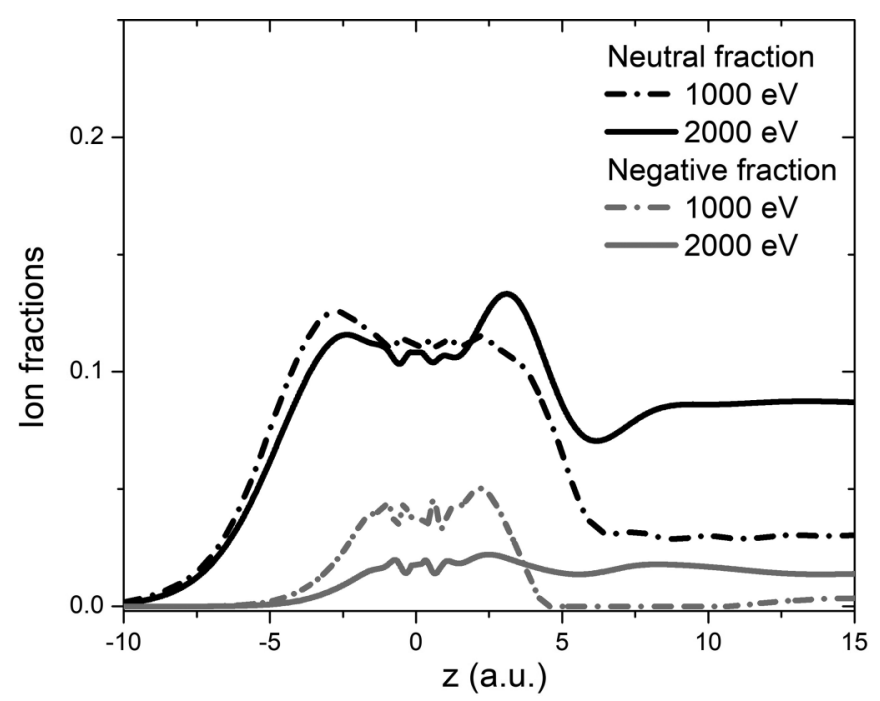

FIG. 13. Neutral (black lines) and negative (gray lines) ion fractions as a function of distance in the case of considering only the $4 s$-band states of the adsorbed potassium, for incoming energies: $1000 \mathrm{eV}$ (short-dashed-dotted line) and $2000 \mathrm{eV}$ (solid line).

process, calculated by removing the $\mathrm{K}$ adatom, but maintaining its position as the scattering center. The large distance of the ion projectile to the carbon atoms is responsible for this very small possibility of a direct charge exchange between them (see the small hydrogen-carbon hopping integrals in Fig. 6).

Figure 13 shows the neutral and negative ion fraction as a function of the distance to the surface, in the case of omitting the K-inner bands. We can conclude, by comparing with the evolution of the charge states along the trajectory shown in Fig. 11, that the $3 p$-band states of adsorbed potassium provides an important source of electrons and that the promotion effect of the $3 s$-core state is favoring the negative charge of the incoming proton.

The conclusions related to the important role of the core $\mathrm{K}-3 p$ state will change for alkaline adsorbates such as $\mathrm{Li}$ and $\mathrm{Na}$, because the core states of both of them are located well below the bottom of the valence band of graphene (the energies referred to the Fermi level are $\mathrm{Li}-1 s=-53.1 \mathrm{eV}$; $\mathrm{Na}-2 p=-28.1 \mathrm{eV}$ and $\mathrm{Na}-2 s=-61.2 \mathrm{eV}$ [33]).

In addition, a memory loss effect is not expected, taking into account that the charge exchange occurs between the atomic state of the projectile and the very narrow bands localized on the adsorbed atom, as reflected in the characteristics of the hydrogen level widths shown in Fig. 7. Furthermore, different final charge fractions were obtained by changing the initial charge state of the hydrogen ion, reaffirming the lack of a memory loss.

Finally, in Fig. 14, we compare the negative $\left(\Gamma^{-}\right)$ and neutral $\left(\Gamma^{0}\right)$ fractions obtained by considering the Coulomb repulsion $U\left(\Gamma^{-}=P^{-}\right.$and $\left.\Gamma^{0}=P^{0}\right)$, with the same quantities constructed from the spinless calculation [26]: $\Gamma^{-}=\left\langle\hat{n}_{a}\right\rangle_{H^{0} \rightarrow H^{-}}\left\langle\hat{n}_{a}\right\rangle_{H^{+} \rightarrow H^{0}}$, the positive ion fraction $\Gamma^{+}=$ $\left(1-\left\langle\hat{n}_{a}\right\rangle_{H^{+} \rightarrow H^{0}}\right)\left(1-\left\langle\hat{n}_{a}\right\rangle_{H^{0} \rightarrow H^{-}}\right)$, and $\Gamma^{0}=1-\Gamma^{+}-\Gamma^{-}$. Here, $\left\langle\hat{n}_{a}\right\rangle_{H^{+} \rightarrow H^{0}}$ means the occupation of the ionization level 


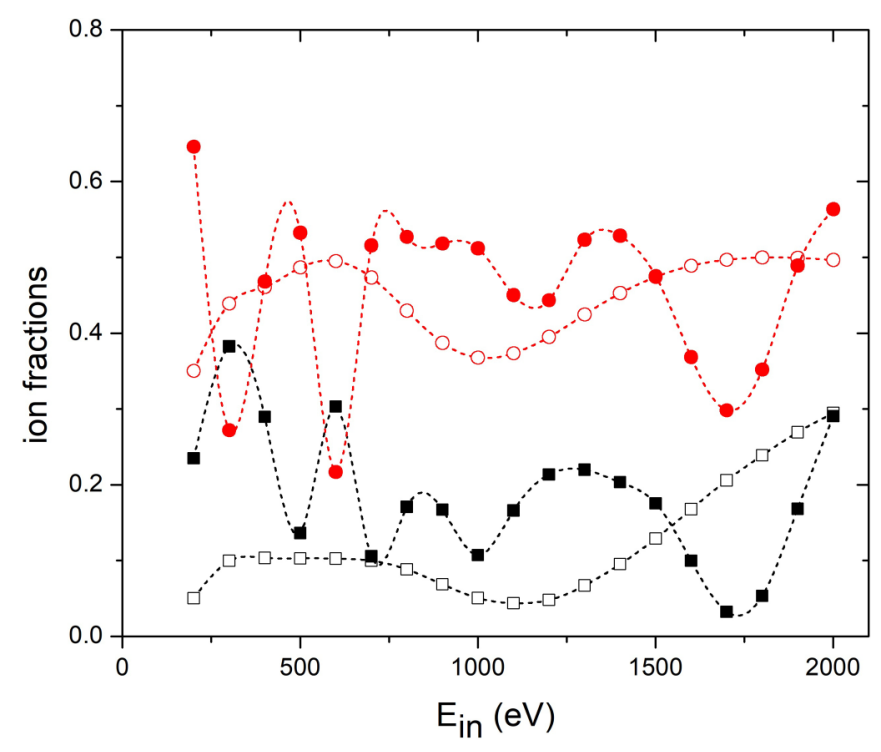

FIG. 14. Ion fractions as a function of the projectile incoming energy calculated by considering (full symbols) and not considering (empty symbols) correlation effects. The squares correspond to negative $\left(\Gamma^{-}\right)$, and circles to neutral $\left(\Gamma^{0}\right)$ fractions.

and $\left\langle\hat{n}_{a}\right\rangle_{H^{0} \rightarrow H^{-}}$, is the occupation of the affinity level (see Sec. III)

We observe from Fig. 14 that the calculation that ignores the correlation effects provides approximately an average of the neutral fraction, while it underestimates the negative ion fraction for energies below $1500 \mathrm{eV}$. But, from the comparison, we extract clearly that the pronounced oscillatory behavior is introduced by the correlated nature of the three possible charge states of hydrogen. Furthermore, the negative hydrogen formation reaches higher values than the measured ones in clean carbon based surfaces such as highly oriented pyrolitic graphite (HOPG) [26,42].

\section{CONCLUSIONS}

In this work we have studied the frontal collision of protons with a potassium atom adsorbed on graphene. Electronic correlation is introduced in the calculation of the LDOS at the adatom site and the charge fractions of the scattered proton.

The local density of states at the $\mathrm{K}$ site includes not only the $4 s$-valence orbital of the $\mathrm{K}$ atom but also the $3 p$-core orbital whose energy resonates with the graphene valence band, and then, it turns out to be widened by the interaction. The $3 s$-core state is also included in the LDOS at the $\mathrm{K}$ site, as an inner band of zero width.

We have taken into account the changes in the LDOS at the $\mathrm{C}$ sites due to the presence of the $\mathrm{K}$ adatom. We found that, in a frontal collision with the $\mathrm{K}$ adatom, the $\mathrm{C}$ atoms practically do not participate in a direct charge exchange process.

The localized features of the spectral density on the $\mathrm{K}$ adatom, intimately related to the band structure of the graphene sheet, enable a full negative ionization during the incoming path and then, in the exit, electron loss takes place, not only in the empty 4s-band but also to the long-lived holes created during the approach to the surface. Afterwards, the charge fluctuation $\mathrm{H}^{0}$ to $\mathrm{H}^{+}$occurs fundamentally at the expense of the holes created in the surface band states during the full negative ionization that took place in the incoming path. There is also a small contribution to the positive ionization process due to the electron loss to the empty K- $4 s$ band, which is habilitated by the more significant level width introduced by the correlation effects.

We found that the $\mathrm{K}-3 p$ band provides an important source of electrons, and that the promotion effect of the K-3s core state is favoring the negative ion formation. The core states of the alkaline atoms adsorbed on graphene will introduce important differences between them: neither Li nor Na have core levels that can resonate with the valence band of graphene.

The pronounced oscillatory behavior of the charge state probabilities with the incoming energy is caused by the correlated states of hydrogen interacting with the localized band states of the potassium adsorbed on graphene. In addition, the negative ion fraction obtained reaches high values compared with the case of hydrogen scattered by $\mathrm{C}$ atoms in carbon based surfaces like HOPG.

Finally, we can conclude that the charge states after the collision with the adsorbed potassium are determined along the whole trajectory, and are dependent on the initial projectile charge state.

\section{ACKNOWLEDGMENTS}

The authors acknowledge financial support by Consejo Nacional de Investigaciones Científicas y Técnicas, Grant No. 0100546, Universidad Nacional del Litoral through the CAI+D Grant, and ANPCyT through Grant No. PICT-20164288.

\section{APPENDIX: FINITE- $U$ APPROXIMATION}

Taking into account the Anderson Hamiltonian introduced in Sec. II B and keeping only terms up to a second order in the surface-atom coupling $V_{\vec{k} a}^{\sigma}(t)$, the equations of motion for the Green functions

$$
\begin{aligned}
F_{\sigma}\left(t, t^{\prime}\right) & =i\left\langle\left[|\sigma\rangle\left\langle\left. 0\right|_{t^{\prime}}, \mid 0\right\rangle\left\langle\left.\sigma\right|_{t}\right]\right\rangle,\right. \\
F_{\uparrow \downarrow}^{\sigma}\left(t, t^{\prime}\right) & =i\left\langle\left[|\uparrow, \downarrow\rangle\left\langle\left.\sigma\right|_{t^{\prime}}, \mid \sigma\right\rangle\left\langle\uparrow,\left.\downarrow\right|_{t}\right]\right\rangle,\right.
\end{aligned}
$$

result in

$$
\begin{aligned}
i \frac{d F_{\sigma}\left(t, t^{\prime}\right)}{d t}= & \varepsilon_{I} F_{\sigma}\left(t, t^{\prime}\right)+i \sum_{\vec{k}} V_{\vec{k} a}^{\sigma}(t)\left[2\left\langle\hat{n}_{\vec{k} \bar{\sigma}}\right\rangle-1\right]\left[\left\langle\hat{c}_{\vec{k} \bar{\sigma}} \mid \bar{\sigma}\right\rangle\langle 0 \mid\rangle e^{i \varepsilon_{\vec{k} \sigma}\left(t^{\prime}-t\right)}+\left\langle\hat{c}_{\vec{k} \bar{\sigma}}^{\dagger} \mid \sigma\right\rangle\langle\uparrow, \downarrow \mid\rangle e^{-i\left(\varepsilon_{\vec{k} \sigma}-E_{2}\right)\left(t^{\prime}-t\right)}\right] \\
& +\int_{t_{0}}^{t^{\prime}} d \tau\left\{\left[\Xi_{\sigma}^{R}(t, \tau)+\Xi_{\bar{\sigma}}^{R<}(t, \tau)\right] F_{\sigma}\left(\tau, t^{\prime}\right)+\left[\Omega_{\sigma}(t, \tau)+\Omega_{\bar{\sigma}}^{<}(t, \tau)\right] G_{\sigma}\left(\tau, t^{\prime}\right)\right\}
\end{aligned}
$$




$$
\begin{aligned}
i \frac{d F_{\uparrow \downarrow}^{\sigma}\left(t, t^{\prime}\right)}{d t}= & \left(\varepsilon_{I}+U\right) F_{\uparrow \downarrow}^{\sigma}\left(t, t^{\prime}\right)+i \sum_{\vec{k}} V_{\vec{k} a}^{\sigma *}(t)\left[2\left\langle\hat{n}_{\vec{k} \vec{\sigma}}\right\rangle-1\right]\left[\langle\mid \uparrow, \downarrow\rangle\left\langle\bar{\sigma} \mid \hat{c}_{\vec{k} \sigma}\right\rangle e^{i \varepsilon_{\vec{k} \sigma}\left(t^{\prime}-t\right)}+\left\langle\hat{c}_{\vec{k} \sigma}^{\dagger} \mid 0\right\rangle\langle\sigma \mid\rangle e^{-i\left(\varepsilon_{\vec{k} \sigma}-E_{2}\right)\left(t^{\prime}-t\right)}\right] \\
& +\int_{t_{0}}^{t^{\prime}} d \tau\left\{\left[\Xi_{\sigma}^{R}(t, \tau)+\Xi_{\sigma}^{R>}(t, \tau)\right] F_{\uparrow \downarrow}^{\sigma}\left(\tau, t^{\prime}\right)+\left[\Omega_{\sigma}(t, \tau)+\Omega_{\sigma}^{>}(t, \tau)\right] G_{\uparrow \downarrow}^{\sigma}\left(\tau, t^{\prime}\right) .\right.
\end{aligned}
$$

For calculating Eqs. (A2) and (A3), it is necessary to calculate the advanced Green functions

$$
\begin{aligned}
G_{\sigma}\left(t, t^{\prime}\right) & =i \Theta\left(t^{\prime}-t\right)\left\langle\left[|\sigma\rangle\left\langle\left. 0\right|_{t^{\prime}}, \mid 0\right\rangle\left\langle\left.\sigma\right|_{t}\right]\right\rangle,\right. \\
G_{\uparrow \downarrow}^{\sigma}\left(t, t^{\prime}\right) & =i \Theta\left(t^{\prime}-t\right)\left\langle\left[|\uparrow, \downarrow\rangle\left\langle\left.\sigma\right|_{t^{\prime}}, \mid \sigma\right\rangle\left\langle\uparrow,\left.\downarrow\right|_{t}\right]\right\rangle,\right.
\end{aligned}
$$

whose motion equations are given by

$$
\begin{aligned}
i \frac{d G_{\sigma}\left(t, t^{\prime}\right)}{d t}= & \delta\left(t^{\prime}-t\right)\langle\mid \sigma\rangle\langle\sigma|+| 0\rangle\langle 0 \mid\rangle+\varepsilon_{I} G_{\sigma}\left(t, t^{\prime}\right) \\
& +i \Theta\left(t^{\prime}-t\right) \sum_{\vec{k}} V_{\vec{k} a}^{\sigma *}(t)\left[\left\langle\hat{c}_{\vec{k} \bar{\sigma}} \mid \bar{\sigma}\right\rangle\langle 0 \mid\rangle e^{i \varepsilon_{\vec{k} \sigma}\left(t^{\prime}-t\right)}-\left\langle\hat{c}_{\vec{k} \bar{\sigma}}^{\dagger} \mid \sigma\right\rangle\langle\uparrow, \downarrow \mid\rangle e^{-i\left(\varepsilon_{\vec{k} \sigma}-E_{2}\right)\left(t^{\prime}-t\right)}\right] \\
& +\int_{t}^{t^{\prime}} d \tau\left[\Xi_{\sigma}(\tau, t)+\Xi_{\bar{\sigma}}^{<}(\tau, t)\right] G_{\sigma}\left(\tau, t^{\prime}\right), \\
i \frac{d G_{\uparrow \downarrow}^{\sigma}\left(t, t^{\prime}\right)}{d t}= & \delta\left(t^{\prime}-t\right)\langle\mid \uparrow, \downarrow\rangle\langle\uparrow, \downarrow|+| \sigma\rangle\langle\sigma \mid\rangle+\left(\varepsilon_{I}+U\right) G_{\uparrow \downarrow}^{\sigma}\left(t, t^{\prime}\right) \\
& +i \Theta\left(t^{\prime}-t\right) \sum_{\vec{k}} V_{\vec{k} a}^{\sigma *}(t)\left[\langle\mid \uparrow, \downarrow\rangle\left\langle\bar{\sigma} \mid \hat{c}_{\vec{k} \sigma}\right\rangle e^{i \varepsilon_{\vec{k} \sigma}\left(t^{\prime}-t\right)}-\left\langle\hat{c}_{\vec{k} \sigma}^{\dagger} \mid 0,0\right\rangle\langle\sigma \mid\rangle e^{-i\left(\varepsilon_{\vec{k} \sigma}-E_{2}\right)\left(t^{\prime}-t\right)}\right] \\
& +\int_{t}^{t^{\prime}} d \tau\left[\Xi_{\sigma}(\tau, t)+\Xi_{\sigma}^{>}(\tau, t)\right] G_{\uparrow \downarrow}^{\sigma}\left(\tau, t^{\prime}\right) .
\end{aligned}
$$

The expressions for the self-energies introduced in Eqs. (A2), (A3), (A5), and (A6), are

$$
\begin{aligned}
& \Xi_{\sigma}(\tau, t)=i \Theta(\tau-t) \sum_{\vec{k}} V_{\vec{k} a}^{\sigma *}(t) V_{\vec{k} a}^{\sigma}(\tau) e^{-i \varepsilon_{\vec{k} \sigma}(t-\tau)}, \\
& \Xi_{\sigma}^{<}(\tau, t)=i \Theta(\tau-t) \sum_{\vec{k}} V_{\vec{k} a}^{\sigma *}(t) V_{\vec{k} a}^{\sigma}(\tau)\left\langle\hat{n}_{\vec{k} \sigma}\right\rangle\left(e^{-i \varepsilon_{\vec{k} \sigma}(t-\tau)}+e^{-i\left(\varepsilon_{\vec{k} \sigma}-E_{2}\right)(\tau-t)}\right), \\
& \Xi_{\sigma}^{>}(\tau, t)=i \Theta(\tau-t) \sum_{\vec{k}} V_{\vec{k} a}^{\sigma *}(t) V_{\vec{k} a}^{\sigma}(\tau)\left\langle 1-\hat{n}_{\vec{k} \sigma}\right\rangle\left(e^{-i \varepsilon_{\vec{k} \sigma}(t-\tau)}+e^{-i\left(\varepsilon_{\vec{k} \sigma}-E_{2}\right)(\tau-t)}\right), \\
& \Omega_{\sigma}^{<}(t, \tau)=i \sum_{\vec{k}} V_{\vec{k} a}^{\sigma}(t)\left[2\left\langle\hat{n}_{\vec{k} \sigma}\right\rangle-1\right]\left\langle\hat{n}_{\vec{k} \sigma}\right\rangle\left[e^{-i \varepsilon_{\vec{k} \sigma}(t-\tau)}-e^{-i\left(\varepsilon_{\vec{k} \sigma}-E_{2}\right)(\tau-t)}\right], \\
& \Omega_{\sigma}^{>}(t, \tau)=i \sum_{\vec{k}} V_{\vec{k} a}^{\sigma}(t)\left[2\left\langle\hat{n}_{\vec{k} \sigma}\right\rangle-1\right]\left\langle 1-\hat{n}_{\vec{k} \sigma}\right\rangle\left[e^{-i \varepsilon_{\vec{k} \sigma}(t-\tau)}-e^{-i\left(\varepsilon_{\vec{k} \sigma}-E_{2}\right)(\tau-t)}\right] .
\end{aligned}
$$

The total energy $E_{2}$ corresponds to the two electron configuration, that is $E_{2}=2 \varepsilon_{I}+U$.

[1] A. J. Bard and L. R. Faulkner, Electrochemical Methods: Fundamentals and Applications (Wiley, New Jersey, 2001).

[2] J. W. Rabalais, Principles and Applications of Ion Scattering Spectrometry (Wiley, New Jersey, 2003).

[3] R. E. Johnson, Energetic Charged-Particle Interactions with Atmospheres and Surfaces (Springer-Verlag, Berlin, 1990).

[4] W. M. Stacey, Fusion Plasma Physics (Wiley-VCH Verlag, Weinheim, 2005).

[5] J. Ishikawa, The Physics and Technology of Ion Sources (WileyVCH Verlag, Weinheim, 2004).
[6] H. H. Brongersma, M. Draxler, M. de Ridder, and P. Bauer, Surf. Sci. Rep. 62, 63 (2007).

[7] H. D. Hagstrum, Phys. Rev. 96, 336 (1954).

[8] J. Los and J. J. C. Geerlings, Phys. Rep. 190, 133 (1990).

[9] H. P. Bonzel, A. M. Bradshaw, and G. Ertl, Physics and Chemistry of Alkali Metal Adsorption (Elsevier, Amsterdam, 1989).

[10] J. N. M. Van Wunnik and J. Los, Phys. Lett. A 87, 288 (1982).

[11] J. N. M. Van Wunnik, J. J. C. Geerlings, E. H. A. Graneman, and J. Los, Surf. Sci. 131, 17 (1983). 
[12] J. J. C. Geerlings, P. W. Van Amersfoort, L. F. Kwakman, E. H. A. Granneman, J. Los, and J. P. Gauyacq, Surf. Sci. 157, 151 (1985).

[13] Y. Yang, Z. Sroubeck, and J. A. Yarmoff, Phys. Rev. B 69, 045420 (2004).

[14] C. B. Weare and J. A. Yarmoff, Surf. Sci. 348, 359 (1996).

[15] C. B. Weare, K. A. H. German, and J. A. Yarmoff, Phys. Rev. B 52, 2066 (1995).

[16] J. J. C. Geerlings, L. F. T. Kwakman, and J. Los, Surf. Sci. 184, 305 (1987).

[17] Q. B. Lu, R. Souda, D. J. O'Connor, and B. V. King, Phys. Rev. Lett. 77, 3236 (1996).

[18] J. A. Yarmof, Y. Yang, and Z. Sroubek, Phys. Rev. Lett. 91, 086104 (2003).

[19] J. P. Gauyacq and A. G. Borisov, J. Phys.: Condens. Matter 10, 6585 (1998).

[20] K. S. Novoselov, V. I. Falko, L. Colombo, P. R. Gellert, M. G. Schwab, and K. Kim, Nature (London) 490, 192 (2012).

[21] K. T. Chan, J. B. Neaton, and M. L. Cohen, Phys. Rev. B 77, 235430 (2008).

[22] N. Dimakis, D. Valdez, F. A. Flor, A. Salgado, K. Adjibi, S. Vargas, and J. Saenz, App. Surf. Sci. 413, 197 (2017).

[23] W. Liu, C. Zhang, M. Deng, and S. Cai, J. Phys. E 93, 265 (2017).

[24] M. A. Romero, A. Iglesias-García, and C. E. Goldberg, Phys. Rev. B 83, 125411 (2011).

[25] E. A. García, A. Iglesias-García, S. C. Gómez Carrillo, and M. A. Romero, App. Surf. Sci. 452, 507 (2018).
[26] R. A. Vidal, F. Bonetto, J. Ferrón, M. A. Romero, E. A. García, and E. C. Goldberg, Surf. Sci. 605, 18 (2011).

[27] M. A. Romero, F. Flores, and E. C. Goldberg, Phys. Rev. B 80, 235427 (2009).

[28] L. V. Keldysh, Zh. Eksp. Teor. Fiz. 47, 1515 (1964).

[29] L. V. Keldysh, Sov. Phys. JETP 20, 1018 (1965).

[30] P. G. Bolcatto, E. C. Goldberg, and M. C. G. Passeggi, Phys. Rev. B 58, 5007 (1998).

[31] P. W. Anderson, Phys. Rev. 124, 41 (1961).

[32] H. C. Hewson, The Kondo Problem to Heavy Fermions (Cambridge University Press, Cambridge, 1993).

[33] M. Cardona and L. Ley, Photoemission in Solids I (SpringerVerlag, New York, 1978).

[34] Z. H. Zhu and G. Q. Lu, Langmuir 20, 10751 (2004).

[35] K. Rytkönen, J. Akola, and M. Manninen, Phys. Rev. B 75, 075401 (2007).

[36] A. Iglesias-García, C. González, and E. C. Goldberg, Phys. Rev. B 96, 075428 (2017).

[37] S. Huzinaga, J. Chem. Phys. 42, 1293 (1965).

[38] S. J. Huzinaga, Gaussian Basis Set for Molecular Calculation (Elsevier, Amsterdam, 1984).

[39] J. P. Lewis, K. R. Glaesemann, G. A. Voth, J. Fritsch, A. A. Demkov, J. Ortega, and O. F. Sankey, Phys. Rev. B 64, 195103 (2001).

[40] P. Jelinek, H. Wang, J. P. Lewis, O. F. Sankey, and J. Ortega, Phys. Rev. B 71, 235101 (2005).

[41] B. Bahrim, S. Yu, B. Makarenko, and J. W. Rabalais, Surf. Sci. 603, 703 (2009).

[42] F. J. Bonetto, M. A. Romero, A. Iglesias-García, R. A. Vidal, and E. C. Goldberg, J. Phys. Chem. C 119, 3124 (2015). 